\title{
REGULARIZATION OF POINT VORTICES FOR THE EULER EQUATION IN DIMENSION TWO
}

\author{
DAOMIN CAO, ZHONGYUAN LIU, AND JUNCHENG WEI
}

\begin{abstract}
In this paper, we construct stationary classical solutions of the incompressible Euler equation approximating singular stationary solutions of this equation. This procedure is carried out by constructing solutions to the following elliptic problem

$$
\begin{cases}-\varepsilon^{2} \Delta u=\sum_{j=1}^{m} \chi_{\Omega_{j}}\left(u-q-\frac{\kappa_{j}}{2 \pi} \ln \frac{1}{\varepsilon}\right)_{+}^{p}, & x \in \Omega, \\ u=0, & x \in \partial \Omega,\end{cases}
$$

where $p>1, \Omega \subset \mathbb{R}^{2}$ is a bounded domain, $\Omega_{i} \subset \subset \Omega, i=1 \cdots, m$ are suitable small domains such that $\Omega_{i} \bigcap \Omega_{j}$ is empty if $i \neq j$ and $q$ is a harmonic function.

We showed that if $\Omega$ is simply-connected smooth domain, then for any given stable critical point of Kirchhoff-Routh function $\mathcal{W}\left(z_{1}, \cdots, z_{m}\right)$ with the positive strength $\kappa_{i}>0$, there is a stationary classical solution approximating stationary $m$ points vortex solution of incompressible Euler equations with vorticity $\sum_{j=1}^{m} \kappa_{i}$.

Existence and asymptotic behavior of single point non-vanishing vortex solutions were studied by D. Smets and J. Van Schaftingen in 22.
\end{abstract}

AMS 2000 Subject Classifications: Primary 35J60; Secondary 35JB05; 35J40

Keywords: The Euler equation; Multiple non-vanishing vortices; Free boundary problem.

\section{INTRODUCTION AND MAIN RESULTS}

The incompressible Euler equations

$$
\left\{\begin{array}{l}
\mathbf{v}_{t}+(\mathbf{v} \cdot \nabla) \mathbf{v}=-\nabla P \\
\nabla \cdot \mathbf{v}=0
\end{array}\right.
$$

describe the evolution of the velocity $\mathbf{v}$ and the pressure $P$ in an incompressible flow. In $\mathbb{R}^{2}$, the vorticity of the flow is defined by $\omega=\nabla \times \mathbf{v}:=\partial_{1} v_{2}-\partial_{2} v_{1}$, which satisfies the equation

$$
\omega_{t}+\mathbf{v} \cdot \nabla \omega=0
$$

Suppose that $\omega$ is known, then the velocity $\mathbf{v}$ can be recovered by Biot-Savart law as following:

$$
\mathbf{v}=\omega * \frac{1}{2 \pi} \frac{-x^{\perp}}{|x|^{2}}
$$


where $x^{\perp}=\left(x_{2},-x_{1}\right)$ if $x=\left(x_{1}, x_{2}\right)$. One special singular solutions of Euler equations is given by $\omega=\sum_{i=1}^{m} \kappa_{i} \delta_{x_{i}(t)}$, which is related

$$
\mathbf{v}=-\sum_{i=1}^{m} \frac{\kappa_{i}}{2 \pi} \frac{\left(x-x_{i}(t)\right)^{\perp}}{\left|x-x_{i}(t)\right|^{2}}
$$

and the positions of the vortices $x_{i}: \mathbb{R} \rightarrow \mathbb{R}^{2}$ satisfy the following Kirchhoff law:

$$
\kappa_{i} \frac{d x_{i}}{d t}=\left(\nabla_{x_{i}} \mathcal{W}\right)^{\perp}
$$

where $\mathcal{W}$ is the so called Kirchhoff-Routh function defined by

$$
\mathcal{W}\left(x_{1}, \cdots, x_{m}\right)=\frac{1}{2} \sum_{i \neq j}^{m} \frac{\kappa_{i} \kappa_{j}}{2 \pi} \log \frac{1}{\left|x_{i}-x_{j}\right|} .
$$

In simply-connected bounded domain $\Omega \subset \mathbb{R}^{2}$, similar singular solutions also exist. Suppose that the normal component of $\mathbf{v}$ vanishes on $\partial \Omega$, then the Kirchhoff-Routh function is

$$
\mathcal{W}\left(x_{1}, \cdots, x_{m}\right)=\frac{1}{2} \sum_{i \neq j}^{m} \kappa_{i} \kappa_{j} G\left(x_{i}, x_{j}\right)+\frac{1}{2} \sum_{i=1}^{m} \kappa_{i}^{2} H\left(x_{i}, x_{i}\right),
$$

where $G$ is the Green function of $-\Delta$ on $\Omega$ with 0 Dirichlet boundary condition and $H$ is its regular part (the Robin function).

Let $v_{n}$ be the outward component of the velocity $\mathbf{v}$ on the boundary $\partial \Omega$, then we see that $\int_{\partial \Omega} v_{n}=0$ due to the fact that $\nabla \cdot \mathbf{v}=0$. Suppose that $\mathbf{v}_{0}$ is the unique harmonic field whose normal component on the boundary $\partial \Omega$ is $v_{n}$, then $\mathbf{v}_{0}$ satisfies

$$
\left\{\begin{array}{l}
\nabla \cdot \mathbf{v}_{0}=0, \text { in } \Omega, \\
\nabla \times \mathbf{v}_{0}=0, \text { in } \Omega, \\
n \cdot \mathbf{v}_{0}=v_{n}, \text { on } \partial \Omega .
\end{array}\right.
$$

If $\Omega$ is simply-connected, then $\mathbf{v}_{0}$ can be written $\mathbf{v}_{0}=\left(\nabla \psi_{0}\right)^{\perp}$, where the stream function $\psi_{0}$ is determined up to a constant by

$$
\left\{\begin{array}{l}
-\Delta \psi_{0}=0, \text { in } \Omega \\
-\frac{\partial \psi_{0}}{\partial \tau}=v_{n}, \text { on } \partial \Omega
\end{array}\right.
$$

where $\frac{\partial \psi_{0}}{\partial \tau}$ denotes the tangential derivative on $\partial \Omega$. The Kirchhoff-Routh function associated to the vortex dynamics becomes(see Lin [17])

$$
\mathcal{W}\left(x_{1}, \cdots, x_{m}\right)=\frac{1}{2} \sum_{i \neq j}^{m} \kappa_{i} \kappa_{j} G\left(x_{i}, x_{j}\right)+\frac{1}{2} \sum_{i=1}^{m} \kappa_{i}^{2} H\left(x_{i}, x_{i}\right)+\sum_{i=1}^{m} \kappa_{i} \psi_{0}\left(x_{i}\right)
$$


It is known that critical points of the Kirchhoff-Routh function $\mathcal{W}$ give rise to stationary vortex points solutions of the Euler equations. As for the existence of critical points of $\mathcal{W}$ given by (1.2), we refer to [5].

Roughly speaking, there are two methods to construct stationary solutions of the Euler equation, which are the vorticity method and the stream-function method. The vorticity method was first established by Arnold and Khesin [3] and further developed by Burton [7] and Turkington [23].

The stream-function method consists in observing that if $\psi$ satisfies $-\Delta \psi=f(\psi)$ for some function $f \in C^{1}(\mathbb{R})$, then $\mathbf{v}=(\nabla \psi)^{\perp}$ and $P=F(\psi)-\frac{1}{2}|\nabla \psi|^{2}$ is a stationary solution to the Euler equations, where $(\nabla \psi)^{\perp}:=\left(\frac{\partial \psi}{\partial x_{2}},-\frac{\partial \psi}{\partial x_{1}}\right), F(t)=\int_{0}^{t} f(s) d s$. Moreover, the velocity $\mathbf{v}$ is irrotational on the set where $f(\psi)=0$.

Set $q=-\psi_{0}$ and $u=\psi-\psi_{0}$, then $u$ satisfies the following boundary value problem

$$
\begin{cases}-\Delta u=f(u-q), & x \in \Omega, \\ u=0, & x \in \partial \Omega .\end{cases}
$$

In addition, if we suppose that $\inf _{\Omega} q>0$ and $f(t)=0, t \leq 0$, the vorticity set $\{x: f(\psi)>$ $0\}$ is bounded away from the boundary.

The motivation to study (1.6) is to justify the weak formulation for point vortex solutions of the incompressible Euler equations by approximating these solutions with classical solutions.

Marchioro and Pulvirenti [19] have approximated these solutions on finite time intervals by considering regularized initial data for the vorticity. On the other hand, the stationary point vortex solutions can also be approximated by stationary classical solutions. See e.g. [6, 21, 22, 23, 24] and the references therein. It is worth pointing out that the above approximations can just give explanation for the formulation to single point vortex solutions. In this paper, we will show that multi-point vortex solutions can be approximated by stationary classical solutions. There are many results for problem (1.6) on the existence and asymptotic behavior of solutions under various assumptions. In [4, 6, 12, 21, 23, the constrained variation methods were used to find solutions for the equation

$$
\begin{cases}-\Delta u=\lambda f(u-q), & x \in \Omega, \\ u=0, & x \in \partial \Omega,\end{cases}
$$

under the constraint $\int_{\Omega} F(u-q)=\mu$, where $\lambda>0$ is a Lagrange multiplier a priori unknown. On the other hand, in [1, 2, 20, 24, 25], the solutions were obtained by using Mountain Pass Lemma for various nonlinearities. For the asymptotic behavior, Berger and Fraenkel [6] began studying the asymptotic behavior for variable $\mu$ and $q$, but the lack of information about $\lambda$ is still an obstacle.

To avoid this obstacle, Yang [24] studied the minimization of the functional $\frac{1}{2} \int_{\Omega}|\nabla u|^{2}-$ $\frac{1}{\varepsilon^{2}} \int_{\Omega} F(u-q)$ under the natural constraint $\int_{\Omega}|\nabla u|^{2}-\frac{1}{\varepsilon^{2}} \int_{\Omega} u f(u-q)=0$ and obtained the asymptotic behavior of the solutions $u_{\varepsilon}$ as $\varepsilon \rightarrow 0$ for $\Omega=\mathbb{R}_{+}^{2}, q(x)=W x_{1}+d$, where $W, d>0$. That is, set $A_{\varepsilon}=\left\{x \in \mathbb{R}_{+}^{2}: f\left(u_{\varepsilon}-q\right)>0\right\}, \kappa_{\varepsilon}=\frac{1}{\varepsilon^{2}} \int_{\Omega} f\left(u_{\varepsilon}-q\right)$ and $x_{\varepsilon} \in A_{\varepsilon}$, then 
$\operatorname{diam} A_{\varepsilon} \rightarrow 0, \operatorname{dist}\left(x_{\varepsilon}, \partial \mathbb{R}_{+}^{2}\right) \rightarrow 0$ and $\frac{u_{\varepsilon}}{\kappa_{\varepsilon}}-G\left(x_{\varepsilon}, \cdot\right) \rightarrow 0$ in $W_{l o c}^{1, r}\left(\mathbb{R}_{+}^{2}\right)$ for $r \in[1,2)$. Later on, similar results were obtained in [15] for bounded domains with additional information that $q\left(x_{\varepsilon}\right) \rightarrow \min _{\Omega} q$. However, it has been pointed out in 22 that the solutions obtained above corresponded to desingularization of point-vortex solutions with vanishing vorticity. To get non-vanishing vortex solutions, D. Smets and J. Van Schaftingen [22] investigated the following problem

$$
\begin{cases}-\varepsilon^{2} \Delta u=\left(u-q-\frac{\kappa}{2 \pi} \ln \frac{1}{\varepsilon}\right)_{+}^{p}, & \text { in } \Omega, \\ u=0, & \text { on } \partial \Omega,\end{cases}
$$

and gave exact asymptotic behavior and expansion of the least energy solution by estimating the upper bounds on the energy. The solutions for (1.9) in [22] were obtained by finding a minimizer of the corresponding functional in a suitable function space, which can only give approximation to a single point non-vanishing vortex. This method is hard to obtain multiple non-vanishing solutions.

In this paper, we approximate stationary vortex solutions of Euler equations (1.1) with multiple non-vanishing vorticity by stationary classical solutions.

Our main result concerning (1.1) is the following:

Theorem 1.1. Suppose that $\Omega \subset \mathbb{R}^{2}$ is a bounded simply-connected smooth domain. Let $v_{n}: \partial \Omega \rightarrow \mathbb{R}$ be such that $v_{n} \in L^{s}(\partial \Omega)$ for some $s>1$ satisfying $\int_{\partial \Omega} v_{n}=0$. Let $\kappa_{i}>0, i=1, \cdots, m$. Then, for any given stable critical point $\left(x_{1}^{*}, \cdots, x_{m}^{*}\right)$ of KirchhoffRouth function $\mathcal{W}\left(x_{1}, \cdots, x_{m}\right)$ defined by (1.5), there exists $\varepsilon_{0}>0$, such that for each $\varepsilon \in\left(0, \varepsilon_{0}\right)$, problem (1.1) has a stationary solution $\mathbf{v}_{\varepsilon}$ with outward boundary flux given by $v_{n}$, such that its vorticities $\omega_{\varepsilon}$ satisfying

$$
\operatorname{supp}\left(\omega_{\varepsilon}\right) \subset \cup_{i=1}^{m} B\left(x_{i, \varepsilon}, C \varepsilon\right) \text { for } x_{i, \varepsilon} \in \Omega, i=1, \cdots, m
$$

and as $\varepsilon \rightarrow 0$

$$
\begin{gathered}
\int_{\Omega} \omega_{\varepsilon} \rightarrow \sum_{i=1}^{m} \kappa_{i}, \\
\left(x_{1, \varepsilon}, \cdots, x_{m, \varepsilon}\right) \rightarrow\left(x_{1}^{*}, \cdots, x_{m}^{*}\right) .
\end{gathered}
$$

Remark 1.2. The simplest case, corresponding to a single point vortex ( $m=1)$ was studied by Smets and Van Schaftingen [22] by minimizing the corresponding energy functional. In their paper $\mathcal{W}\left(x_{1, \varepsilon}\right) \rightarrow \sup _{x \in \Omega} \mathcal{W}(x)$. Even in the case $m=1$, our result extends theirs to general critical points (with additional assumption that the critical point is nondegenerate). The method used in [22] can not be applied to deal with general critical point cases. The method used here is constructive and is completely different from theirs.

Remark 1.3. In this case that $m=1$ suppose that $x_{1}$ is a strict local maximum(or minimum) point of Kirchhoff-Routh function $\mathcal{W}(x)$ defined by (1.5), statement of Theorem 1.1 still holds which can be proved similarly(see Remark 1.51). Thus we can obtain corresponding existence result in [22]. 
Theorem 1.1 is proved via considering the following problem

$$
\begin{cases}-\varepsilon^{2} \Delta u=\sum_{j=1}^{m} \chi_{\Omega_{j}}\left(u-q-\frac{\kappa_{j}}{2 \pi} \ln \frac{1}{\varepsilon}\right)_{+}^{p}, & x \in \Omega, \\ u=0, & x \in \partial \Omega,\end{cases}
$$

where $p>1, q \in C^{2}(\Omega), \Omega \subset \mathbb{R}^{2}$ is a bounded domain, $\Omega_{j} \subset \Omega$ is a subdomain such that $x_{j}^{*} \in \Omega_{j}, j=1, \cdots, m$ and $\Omega_{i} \cap \Omega_{j}=\varnothing$ if $i \neq j$.

Theorem 1.4. Suppose $q \in C^{2}(\Omega)$. For $\kappa_{i}>0, i=1, \cdots, m$. Then, for any given $C^{1}$-stable critical point $\left(x_{1}^{*}, \cdots, x_{m}^{*}\right)$ of Kirchhoff-Routh function $\mathcal{W}\left(x_{1}, \cdots, x_{m}\right)$ defined by (1.5), there exists $\varepsilon_{0}>0$, such that for each $\varepsilon \in\left(0, \varepsilon_{0}\right)$, (1.9) has a solution $u_{\varepsilon}$, such that the set $\Omega_{\varepsilon, i}=\left\{x: u_{\varepsilon}(x)-\frac{\kappa_{i}}{2 \pi} \ln \frac{1}{\varepsilon}-q(x)>0\right\} \subset \subset \Omega_{i}, i=1,2, \cdots, m$ and each $\Omega_{\varepsilon, i}$ shrinks to $x_{i}^{*} \in \Omega$, as $\varepsilon \rightarrow 0$.

Remark 1.5. For the case $m=1$ suppose that $x_{1}$ is a strict local maximum(or minimum) point of Kirchhoff-Routh function $\mathcal{W}(x)$ defined by (1.5), statement of Theorem 1.4 still holds which can be proved by making corresponding modification of the proof of Theorem 1.4 in obtaining critical point of $K(z)$ defined by (4.1) (see Propositions 2.3,2.5 and 2.6 [9] for detailed arguments).

For domains which may not be simply-connected, we show in the following result that the topology of the domain plays an important role in the existence of solutions.

Theorem 1.6. Suppose that the homology of $\Omega$ is nontrivial. Then, for any positive integer $m$, there exists $\varepsilon_{0}>0$, such that for each $\varepsilon \in\left(0, \varepsilon_{0}\right)$, (1.9) has a solution $u_{\varepsilon}$, such that the set $\Omega_{\varepsilon, i}=\left\{x: u_{\varepsilon}(x)-\frac{\kappa_{i}}{2 \pi} \ln \frac{1}{\varepsilon}-q(x)>0\right\} \subset \subset \Omega_{i}, i=1,2, \cdots, m$ and each $\Omega_{\varepsilon, i}$ shrinks to a point $x_{i}^{*} \in \Omega$, as $\varepsilon \rightarrow 0$. Moreover $x_{i}^{*} \neq x_{j}^{*}$, if $i \neq j$.

Remark 1.7. Since $m$ is arbitrary, from Theorem 1.6, we can see that the number of solutions for (1.10) is unbounded as $\delta \rightarrow 0$.

Not as in [22] where (1.9) is investigated directly, we prove Theorem 1.1, Theorem 1.4 and Theorem 1.6 by considering an equivalent problem of (1.9) instead. Let $w=\frac{2 \pi}{|\ln \varepsilon|} u$ and $\delta=\varepsilon\left(\frac{2 \pi}{|\ln \varepsilon|}\right)^{\frac{p-1}{2}}$, then (1.9) becomes

$$
\begin{cases}-\delta^{2} \Delta w=\sum_{j=1}^{m} \chi_{\Omega_{j}}\left(w-\kappa_{j}-\frac{2 \pi}{|\ln \varepsilon|} q(x)\right)_{+}^{p}, & \text { in } \Omega \\ w=0, & \text { on } \partial \Omega .\end{cases}
$$

We will use a reduction argument to prove Theorem 1.4 and Theorem 1.6. To this end, we need to construct an approximate solution for (1.10). For the problem studied in this paper, the corresponding "limit" problem in $\mathbb{R}^{2}$ has no bounded nontrivial solution. So, we will follow the method in [10, 11] to construct an approximate solution. Since there are two parameters $\delta, \varepsilon$ in problem (1.10), which causes some difficulty, we must take this influence into careful consideration and give delicate estimates in order to perform the reduction argument. For example we need to consider $\left(s_{1, \delta}, \cdots, s_{m, \delta}\right)$ and $\left(a_{1, \delta}, \cdots, a_{m, \delta}\right)$ together in Lemma 2.1. 
As a final remark, we point out that problem (1.10) can be considered as a free boundary problem. Similar problems have been studied extensively. The reader can refer to [8, 10, 11, 13, 16] for more results on this kind of problem.

This paper is organized as follows. In section 2, we construct the approximate solution for (1.10). We will carry out a reduction argument in section 3 and the main results will be proved in section 4 . We put some basic estimates in the appendix.

\section{Approximate solutions}

In the section, we will construct approximate solutions for (1.10).

Let $R>0$ be a large constant, such that for any $x \in \Omega, \Omega \subset \subset B_{R}(x)$. Consider the following problem:

$$
\begin{cases}-\delta^{2} \Delta w=(w-a)_{+}^{p}, & \text { in } B_{R}(0) \\ w=0, & \text { on } \partial B_{R}(0)\end{cases}
$$

where $a>0$ is a constant. Then, (2.1) has a unique solution $W_{\delta, a}$, which can be written as

$$
W_{\delta, a}(x)= \begin{cases}a+\delta^{2 /(p-1)} s_{\delta}^{-2 /(p-1)} \phi\left(\frac{|x|}{s_{\delta}}\right), & |x| \leq s_{\delta} \\ a \ln \frac{|x|}{R} / \ln \frac{s_{\delta}}{R}, & s_{\delta} \leq|x| \leq R,\end{cases}
$$

where $\phi(x)=\phi(|x|)$ is the unique solution of

$$
-\Delta \phi=\phi^{p}, \quad \phi>0, \phi \in H_{0}^{1}\left(B_{1}(0)\right)
$$

and $s_{\delta} \in(0, R)$ satisfies

which implies

$$
\delta^{2 /(p-1)} s_{\delta}^{-2 /(p-1)} \phi^{\prime}(1)=\frac{a}{\ln \left(s_{\delta} / R\right)}
$$

$$
\frac{s_{\delta}}{\delta|\ln \delta|^{(p-1) / 2}} \rightarrow\left(\frac{\left|\phi^{\prime}(1)\right|}{a}\right)^{(p-1) / 2}>0, \quad \text { as } \delta \rightarrow 0 .
$$

Moreover, by Pohozaev identity, we can get that

$$
\int_{B_{1}(0)} \phi^{p+1}=\frac{\pi(p+1)}{2}\left|\phi^{\prime}(1)\right|^{2} \text { and } \int_{B_{1}(0)} \phi^{p}=2 \pi\left|\phi^{\prime}(1)\right| .
$$

For any $z \in \Omega$, define $W_{\delta, z, a}(x)=W_{\delta, a}(x-z)$. Because $W_{\delta, z, a}$ does not vanish on $\partial \Omega$, we need to make a projection. Let $P W_{\delta, z, a}$ be the solution of

$$
\begin{cases}-\delta^{2} \Delta w=\left(W_{\delta, z, a}-a\right)_{+}^{p}, & \text { in } \Omega \\ w=0, & \text { on } \partial \Omega\end{cases}
$$

Then

$$
P W_{\delta, z, a}=W_{\delta, z, a}-\frac{a}{\ln \frac{R}{s_{\delta}}} g(x, z)
$$

where $g(x, z)$ satisfies 


$$
\begin{cases}-\Delta g=0, & \text { in } \Omega \\ g=\ln \frac{R}{|x-z|}, & \text { on } \partial \Omega\end{cases}
$$

It is easy to see that

$$
g(x, z)=\ln R+2 \pi h(x, z),
$$

where $h(x, z)=-H(x, z)$.

We will construct solutions for (1.10) of the form

$$
\sum_{j=1}^{m} P W_{\delta, z_{j}, a_{\delta, j}}+\omega_{\delta}
$$

where $z_{j} \in \Omega$ for $j=1, \cdots, m, \omega_{\delta}$ is a perturbation term. To obtain a good estimate for $\omega_{\delta}$, we need to choose $a_{\delta, j}$ properly.

By (2.3), we have

$$
\begin{aligned}
& -\delta^{2} \Delta \sum_{j=1}^{m} P W_{\delta, z_{j}, a_{\delta, j}}-\sum_{j=1}^{m} \chi_{\Omega_{j}}\left(\sum_{i=1}^{m} P W_{\delta, z_{i}, a_{\delta, i}}-\kappa_{j}-\frac{2 \pi q}{|\ln \varepsilon|}\right)_{+}^{p} \\
= & \sum_{j=1}^{m}\left(W_{\delta, z_{j}, a_{\delta, j}}-a_{\delta, j}\right)_{+}^{p}-\sum_{j=1}^{m} \chi_{\Omega_{j}}\left(\sum_{i=1}^{m}\left(W_{\delta, z_{i}, a_{\delta, i}}-\frac{a_{\delta, i}}{\ln \frac{R}{s_{\delta}}} g\left(y, z_{i}\right)\right)-\kappa_{j}-\frac{2 \pi q}{|\ln \varepsilon|}\right)_{+}^{p} .
\end{aligned}
$$

Denote $Z=\left(z_{1}, \cdots, z_{m}\right) \in \mathbb{R}^{2 m}$. In this paper, we always assume that $z_{j} \in \Omega$ satisfies

$$
d\left(z_{j}, \partial \Omega\right) \geq \varrho>0, \quad\left|z_{i}-z_{j}\right| \geq \varrho^{\bar{L}}, \quad i, j=1, \cdots, m, i \neq j,
$$

where $\varrho>0$ is a fixed small constant and $\bar{L}>0$ is a fixed large constant.

Lemma 2.1. For $\delta>0$ small, there exist $\left(s_{\delta, 1}(Z), \cdots, s_{\delta, m}(Z)\right)$ and $\left(a_{\delta, 1}(Z), \cdots, a_{\delta, m}(Z)\right)$ satisfying the following system

$$
\delta^{2 /(p-1)} s_{i}^{-2 /(p-1)} \phi^{\prime}(1)=\frac{a_{i}}{\ln \left(s_{i} / R\right)}, \quad i=1, \cdots, m
$$

and

$$
a_{i}=\kappa_{i}+\frac{2 \pi q\left(z_{i}\right)}{|\ln \varepsilon|}+\frac{g\left(z_{i}, z_{i}\right)}{\ln \frac{R}{s_{i}}} a_{i}-\sum_{j \neq i}^{m} \frac{\bar{G}\left(z_{i}, z_{j}\right)}{\ln \frac{R}{s_{j}}} a_{j}, \quad i=1, \cdots, m
$$

where for $i=1, \cdots, m$,

$$
\bar{G}\left(x, z_{i}\right)=\ln \frac{R}{\left|x-z_{i}\right|}-g\left(x, z_{i}\right)
$$


Proof. We will show that system (2.6)-(2.7) has a solution $\left(s_{1}, \cdots, s_{m}, a_{1}, \cdots, a_{m}\right)$ in $\mathcal{D} \doteq$ $\left[\frac{\delta}{|\ln \delta|}, \delta|\ln \delta|\right]^{m} \times \prod_{i=1}^{m}\left[\frac{\kappa_{i}}{2}, \frac{3 \kappa_{i}}{2}\right]$. It is easy to see, for fixed $\left(s_{1}, \cdots, s_{m}\right)$ with $0<\delta<\delta^{*}$ small, that (2.7) has a solution $\left(a_{1}, \cdots, a_{m}\right)$ depending on $\left(s_{1}, \cdots, s_{m}\right)$, such that $\frac{\kappa_{i}}{2} \leq a_{i} \leq \frac{3 \kappa_{i}}{2}$. For such $\left(a_{1}, \cdots, a_{m}\right)$ and for $i=1, \cdots, m$ define

$$
\theta_{i}\left(s_{1}, \cdots, s_{m}\right)=\frac{s_{i}^{\frac{2}{p-1}}}{\ln \frac{R}{s_{i}}}+\frac{\phi^{\prime}(1)}{a_{i}} \delta^{\frac{2}{p-1}}
$$

then it is easy to verify that

$$
\left\{\begin{array}{cl}
\theta_{i}\left(s_{1}, \cdots, s_{m}\right)>0, & s_{i}=\delta|\ln \delta|, s_{j} \in\left[\frac{\delta}{|\ln \delta|}, \delta|\ln \delta|\right] \text { for } j=1, \cdots, m, j \neq i \\
\theta_{i}\left(s_{1}, \cdots, s_{m}\right)<0, & s_{i}=\frac{\delta}{|\ln \delta|}, s_{j} \in\left[\frac{\delta}{|\ln \delta|}, \delta|\ln \delta|\right] \text { for } j=1, \cdots, m, j \neq i
\end{array}\right.
$$

By the Poincaré-Miranda Theorem in [14, 18], we can get $\left(s_{\delta, 1}, \cdots, s_{\delta, m}\right)$ such that $\theta_{i}\left(s_{\delta, 1}, \cdots, s_{\delta, m}\right)=0$. Therefore we have completed our proof of Lemma 2.1.

For simplicity, for given $Z=\left(z_{1}, \cdots, z_{m}\right)$, in this paper, we will use $a_{\delta, i}, s_{\delta, i}$ instead of $a_{\delta, i}(Z), s_{\delta, i}(Z)$.

Remark 2.2. More precisely, we have the following relation

$$
\begin{gathered}
\frac{1}{\ln \frac{R}{s_{\delta, i}}}=\frac{1}{\ln \frac{R}{\varepsilon}}+O\left(\frac{\ln |\ln \varepsilon|}{|\ln \varepsilon|^{2}}\right), i=1, \cdots, m, \\
a_{\delta, i}=\kappa_{i}+\frac{2 \pi q\left(z_{i}\right)}{|\ln \varepsilon|}+\frac{g\left(z_{i}, z_{i}\right)}{\ln \frac{R}{\varepsilon}}-\sum_{j \neq i}^{m} \frac{\bar{G}\left(z_{i}, z_{j}\right)}{\ln \frac{R}{\varepsilon}}+O\left(\frac{\ln |\ln \varepsilon|}{|\ln \varepsilon|^{2}}\right), i=1, \cdots, m, \\
\frac{\partial a_{\delta, i}}{\partial z_{j, h}}=O\left(\frac{1}{|\ln \varepsilon|}\right), \frac{\partial s_{\delta, i}}{\partial z_{j, h}}=O\left(\frac{\varepsilon}{|\ln \varepsilon|}\right), i, j=1, \cdots, m, h=1,2 .
\end{gathered}
$$

Indeed, (2.8) can be deduced from (2.6) (see [11], for example). (2.9) can been deduced from (2.8) and (2.7). Differentiating both sides of (2.6) and (2.7) with respect to $z_{j, h}$ we can get a linear system of $\frac{\partial a_{\delta, i}}{\partial z_{j, h}}$ and $\frac{\partial s_{\delta, i}}{\partial z_{j, h}}$, which will deduces (2.10).

From now on we will always choose $\left(a_{\delta, 1}, \cdots, a_{\delta, m}\right)$ and $\left(s_{\delta, 1}, \cdots, s_{\delta, m}\right)$ such that (2.6) and (2.7) are satisfied. For $\left(a_{\delta, 1}, \cdots, a_{\delta, m}\right)$ and $\left(s_{\delta, 1}, \cdots, s_{\delta, m}\right)$ chosen in such a way define

$$
P_{\delta, Z, j}=P W_{\delta, z_{j}, a_{\delta, j}}, P_{\delta, Z}=\sum_{j=1}^{m} P_{\delta, Z, j}
$$

Then, we find that for $x \in B_{L s_{\delta, i}}\left(z_{i}\right)$, where $L>0$ is any fixed constant, 


$$
\begin{aligned}
& P_{\delta, Z, i}(x)-\kappa_{i}-\frac{2 \pi q(x)}{|\ln \varepsilon|}=W_{\delta, z_{i}, a_{\delta, i}}(x)-\frac{a_{\delta, i}}{\ln \frac{R}{s_{\delta, i}}} g\left(x, z_{i}\right)-\kappa_{i}-\frac{2 \pi q(x)}{|\ln \varepsilon|} \\
= & W_{\delta, z_{i}, a_{\delta, i}}(x)-\kappa_{i}-\frac{a_{\delta, i}}{\ln \frac{R}{s_{\delta, i}}} g\left(z_{i}, z_{i}\right)-\frac{a_{\delta, i}}{\ln \frac{R}{s_{\delta, i}}}\left(\left\langle D g\left(z_{i}, z_{i}\right), x-z_{i}\right\rangle+O\left(\left|x-z_{i}\right|^{2}\right)\right) \\
& -\frac{2 \pi q\left(z_{i}\right)}{|\ln \varepsilon|}-\frac{2 \pi}{|\ln \varepsilon|}\left(\left\langle D q\left(z_{i}\right), x-z_{i}\right\rangle+O\left(\left|x-z_{i}\right|^{2}\right)\right) \\
= & W_{\delta, z_{i}, a_{\delta, i}}(x)-\kappa_{i}-\frac{2 \pi q\left(z_{i}\right)}{|\ln \varepsilon|}-\frac{2 \pi}{|\ln \varepsilon|}\left\langle D q\left(z_{i}\right), x-z_{i}\right\rangle \\
& -\frac{a_{\delta, i}}{\ln \frac{R}{s_{\delta, i}}} g\left(z_{i}, z_{i}\right)-\frac{a_{\delta, i}}{\ln \frac{R}{s_{\delta, i}}}\left\langle D g\left(z_{i}, z_{i}\right), x-z_{i}\right\rangle+O\left(\frac{s_{\delta, i}^{2}}{|\ln \varepsilon|}\right),
\end{aligned}
$$

and for $j \neq i$ and $x \in B_{L s_{\delta, i}}\left(z_{i}\right)$, by (2.2)

$$
\begin{aligned}
& P_{\delta, Z, j}(x)=W_{\delta, z_{j}, a_{\delta, j}}(x)-\frac{a_{\delta, j}}{\ln \frac{R}{s_{\delta, j}}} g\left(x, z_{j}\right)=\frac{a_{\delta, j}}{\ln \frac{R}{s_{\delta, j}}} \bar{G}\left(x, z_{j}\right) \\
= & \frac{a_{\delta, j}}{\ln \frac{R}{s_{\delta, j}}} \bar{G}\left(z_{i}, z_{j}\right)+\frac{a_{\delta, j}}{\ln \frac{R}{s_{\delta, j}}}\left\langle D \bar{G}\left(z_{i}, z_{j}\right), x-z_{i}\right\rangle+O\left(\frac{s_{\delta, i}^{2}}{|\ln \varepsilon|}\right) .
\end{aligned}
$$

So, by using (2.7), we obtain

$$
\begin{aligned}
& P_{\delta, Z}(x)-\kappa_{i}-\frac{2 \pi q(x)}{|\ln \varepsilon|} \\
= & W_{\delta, z_{i}, a_{\delta, i}}(x)-a_{\delta, i}-\frac{2 \pi}{|\ln \varepsilon|}\left\langle D q\left(z_{i}\right), x-z_{i}\right\rangle-\frac{a_{\delta, i}}{\ln \frac{R}{s_{\delta, i}}}\left\langle D g\left(z_{i}, z_{i}\right), x-z_{i}\right\rangle \\
& +\sum_{j \neq i}^{m} \frac{a_{\delta, j}}{\ln \frac{R}{s_{\delta, j}}}\left\langle D \bar{G}\left(z_{i}, z_{j}\right), x-z_{i}\right\rangle+O\left(\frac{s_{\delta, i}^{2}}{|\ln \varepsilon|}\right), \quad x \in B_{L s_{\delta, i}}\left(z_{i}\right) .
\end{aligned}
$$

We end this section by giving the following formula which can be obtained by direct computation and will be used in the next two sections.

$$
\begin{aligned}
& \frac{\partial W_{\delta, z_{i}, a_{\delta, i}}(x)}{\partial z_{i, h}} \\
& = \begin{cases}\frac{1}{\delta}\left(\frac{a_{\delta, i}}{\left|\phi^{\prime}(1)\right|\left|\ln \frac{R}{s_{\delta, i}}\right|}\right)^{(p+1) / 2} \phi^{\prime}\left(\frac{\left|x-z_{i}\right|}{s_{\delta, i}}\right) \frac{z_{i, h}-x_{h}}{\left|x-z_{i}\right|}+O\left(\frac{1}{|\ln \varepsilon|}\right), & x \in B_{s_{\delta, i}}\left(z_{i}\right), \\
-\frac{a_{\delta, i}}{\ln \frac{R}{s_{\delta, i}}} \frac{z_{i, h}-x_{h}}{\left|x-z_{i}\right|^{2}}+O\left(\frac{1}{|\ln \varepsilon|}\right), & x \in \Omega \backslash B_{s_{\delta, i}}\left(z_{i}\right) .\end{cases}
\end{aligned}
$$




\section{THE REDUCTION}

Let

$$
w(x)= \begin{cases}\phi(|x|), & |x| \leq 1 \\ \phi^{\prime}(1) \ln |x|, & |x|>1\end{cases}
$$

Then $w \in C^{1}\left(\mathbb{R}^{2}\right)$. Since $\phi^{\prime}(1)<0$ and $\ln |x|$ is harmonic for $|x|>1$, we see that $w$ satisfies

$$
-\Delta w=w_{+}^{p}, \quad \text { in } \mathbb{R}^{2} .
$$

Moreover, since $w_{+}$is Lip-continuous, by the Schauder estimate, $w \in C^{2, \alpha}$ for any $\alpha \in$ $(0,1)$.

Consider the following problem:

$$
-\Delta v-p w_{+}^{p-1} v=0, \quad v \in L^{\infty}\left(\mathbb{R}^{2}\right)
$$

It is easy to see that $\frac{\partial w}{\partial x_{i}}, i=1,2$, is a solution of (3.2). Moreover, from Dancer and Yan [11], we know that $w$ is also non-degenerate, in the sense that the kernel of the operator $L v:=-\Delta v-p w_{+}^{p-1} v, v \in D^{1,2}\left(\mathbb{R}^{2}\right)$ is spanned by $\left\{\frac{\partial w}{\partial x_{1}}, \frac{\partial w}{\partial x_{2}}\right\}$.

Recall that $Z=\left(z_{1}, \cdots, z_{m}\right)$, and $z_{j} \in \Omega$ satisfies

$$
d\left(z_{j}, \partial \Omega\right) \geq \varrho>0, \quad\left|z_{i}-z_{j}\right| \geq \varrho^{\bar{L}}, \quad i \neq j,
$$

where $\varrho>0$ is a fixed small constant, and $\bar{L}>0$ is a large constant.

Let $P_{\delta, Z, j}$ be the function defined in (2.11). Set

and

$$
F_{\delta, Z}=\left\{u: u \in L^{p}(\Omega), \int_{\Omega} \frac{\partial P_{\delta, Z, j}}{\partial z_{j, h}} u=0, j=1, \cdots, m, h=1,2\right\},
$$

$$
E_{\delta, Z}=\left\{u: u \in W^{2, p}(\Omega) \cap H_{0}^{1}(\Omega), \int_{\Omega} \Delta\left(\frac{\partial P_{\delta, Z, j}}{\partial z_{j, h}}\right) u=0, j=1, \cdots, m, h=1,2\right\} .
$$

For any $u \in L^{p}(\Omega)$, define $Q_{\delta} u$ as follows:

$$
Q_{\delta} u=u-\sum_{j=1}^{m} \sum_{h=1}^{2} b_{j, h}\left(-\delta^{2} \Delta\left(\frac{\partial P_{\delta, Z, j}}{\partial z_{j, h}}\right)\right)
$$

where the constants $b_{j, h}, j=1, \cdots, m, h=1,2$, satisfy

$$
\sum_{j=1}^{m} \sum_{h=1}^{2} b_{j, h}\left(-\delta^{2} \int_{\Omega} \Delta\left(\frac{\partial P_{\delta, Z, j}}{\partial z_{j, h}}\right) \frac{\partial P_{\delta, Z, i}}{\partial z_{i, h}}\right)=\int_{\Omega} u \frac{\partial P_{\delta, Z, i}}{\partial z_{i, h}} .
$$

Since $\int_{\Omega} \frac{\partial P_{\delta, Z, j}}{\partial z_{j, h}} Q_{\delta} u=0$, the operator $Q_{\delta}$ can be regarded as a projection from $L^{p}(\Omega)$ to $F_{\delta, Z}$. In order to show that we can solve (3.4) to obtain $b_{j, h}$, we just need the following estimate ( by (2.10) and (2.13) $)$ : 


$$
\begin{aligned}
& -\delta^{2} \int_{\Omega} \Delta\left(\frac{\partial P_{\delta, Z, j}}{\partial z_{j, h}}\right) \frac{\partial P_{\delta, Z, i}}{\partial z_{i, \bar{h}}} \\
= & p \int_{\Omega}\left(W_{\delta, z_{j}, a_{\delta, j}}-a_{\delta, j}\right)_{+}^{p-1}\left(\frac{\partial W_{\delta, z_{j}, a_{\delta, j}}}{\partial z_{j, h}}-\frac{\partial a_{\delta, j}}{\partial z_{j, h}}\right) \frac{\partial P_{\delta, Z, i}}{\partial z_{i, \bar{h}}} \\
= & \delta_{i j h \bar{h}} \frac{c}{|\ln \varepsilon|^{p+1}}+O\left(\frac{\varepsilon}{|\ln \varepsilon|^{p+1}}\right)
\end{aligned}
$$

where $c>0$ is a constant, $\delta_{i j h \bar{h}}=1$, if $i=j$ and $h=\bar{h}$; otherwise, $\delta_{i j h \bar{h}}=0$.

Set

$$
L_{\delta} u=-\delta^{2} \Delta u-\sum_{j=1}^{m} p \chi_{\Omega_{j}}\left(P_{\delta, Z}-\kappa_{j}-\frac{2 \pi q(x)}{|\ln \varepsilon|}\right)_{+}^{p-1} u .
$$

We have the following lemma.

Lemma 3.1. There are constants $\rho_{0}>0$ and $\delta_{0}>0$, such that for any $\delta \in\left(0, \delta_{0}\right], Z$ satisfying (3.3), $u \in E_{\delta, Z}$ with $Q_{\delta} L_{\delta} u=0$ in $\Omega \backslash \cup_{j=1}^{m} B_{L s_{\delta, j}}\left(z_{j}\right)$ for some $L>0$ large, then

$$
\left\|Q_{\delta} L_{\delta} u\right\|_{L^{p}(\Omega)} \geq \frac{\rho_{0} \delta^{\frac{2}{p}}}{|\ln \delta|^{\frac{(p-1)^{2}}{p}}}\|u\|_{L^{\infty}(\Omega)} .
$$

Proof. Set $s_{n, j}=s_{\delta_{n}, j}$. We will use $\|\cdot\|_{p},\|\cdot\|_{\infty}$ to denote $\|\cdot\|_{L^{p}(\Omega)}$ and $\|\cdot\|_{L^{\infty}(\Omega)}$ respectively.

We argue by contradiction. Suppose that there are $\delta_{n} \rightarrow 0, Z_{n}$ satisfying (3.3) and $u_{n} \in E_{\delta_{n}, Z_{n}}$ with $Q_{\delta_{n}} L_{\delta_{n}} u_{n}=0$ in $\Omega \backslash \cup_{j=1}^{m} B_{L s_{n, j}}\left(z_{j, n}\right),\left\|u_{n}\right\|_{\infty}=1$, such that

$$
\left\|Q_{\delta_{n}} L_{\delta_{n}} u_{n}\right\|_{p} \leq \frac{1}{n} \frac{\delta_{n}^{\frac{2}{p}}}{\left|\ln \delta_{n}\right|^{\frac{(p-1)^{2}}{p}}}
$$

Firstly, we estimate $b_{j, h, n}$ in the following formula:

$$
Q_{\delta_{n}} L_{\delta_{n}} u_{n}=L_{\delta_{n}} u_{n}-\sum_{j=1}^{m} \sum_{h=1}^{2} b_{j, h, n}\left(-\delta_{n}^{2} \Delta \frac{\partial P_{\delta_{n}, Z_{n}, j}}{\partial z_{j, h}}\right)
$$

For each fixed $i$, multiplying (3.6) by $\frac{\partial P_{\delta_{n}, Z_{n}, i}}{\partial z_{i, \bar{h}}}$, noting that

$$
\int_{\Omega}\left(Q_{\delta_{n}} L_{\delta_{n}} u_{n}\right) \frac{\partial P_{\delta_{n}, Z_{n}, i}}{\partial z_{i, \bar{h}}}=0
$$

we obtain 


$$
\begin{aligned}
& \int_{\Omega} u_{n} L_{\delta_{n}}\left(\frac{\partial P_{\delta_{n}, Z_{n}, i}}{\partial z_{i, \bar{h}}}\right)=\int_{\Omega}\left(L_{\delta_{n}} u_{n}\right) \frac{\partial P_{\delta_{n}, Z_{n}, i}}{\partial z_{i, h}} \\
& =\sum_{j=1}^{m} \sum_{\bar{h}=1}^{2} b_{j, h, n} \int_{\Omega}\left(-\delta_{n}^{2} \Delta \frac{\partial P_{\delta_{n}, Z_{n}, j}}{\partial z_{j, h}}\right) \frac{\partial P_{\delta_{n}, Z_{n}, i}}{\partial z_{i, h}}
\end{aligned}
$$

Using (2.12) and Lemma A.1, we obtain

$$
\begin{aligned}
& \int_{\Omega} u_{n} L_{\delta_{n}}\left(\frac{\partial P_{\delta_{n}, Z_{n}, i}}{\partial z_{i, \bar{h}}}\right) \\
& =\int_{\Omega}\left(-\delta_{n}^{2} \Delta\left(\frac{\partial P_{\delta_{n}, Z_{n}, i}}{\partial z_{i, \bar{h}}}\right)-\sum_{j=1}^{m} p \chi_{\Omega_{j}}\left(P_{\delta_{n}, Z_{n}}-\kappa_{j}-\frac{2 \pi q(x)}{\left|\ln \varepsilon_{n}\right|}\right)_{+}^{p-1} \frac{\partial P_{\delta_{n}, Z_{n}, i}}{\partial z_{i, \bar{h}}}\right) u_{n} \\
& =p \int_{\Omega}\left(W_{\delta_{n}, z_{i, n}, a_{\delta_{n}, i}}-a_{\delta_{n}, i}\right)_{+}^{p-1}\left(\frac{\partial W_{\delta_{n}, z_{i, n}, a_{\delta_{n}, i}}}{\partial z_{i, h}}-\frac{\partial a_{\delta_{n}, i}}{\partial z_{i, \bar{h}}}\right) u_{n} \\
& -\sum_{j=1}^{m} p \int_{\Omega_{j}}\left(W_{\delta_{n}, z_{j, n}, a_{\delta_{n}, j}}-a_{\delta_{n}, j}+O\left(\frac{s_{n, j}}{\left|\ln \varepsilon_{n}\right|}\right)\right)_{+}^{p-1} \frac{\partial P_{\delta_{n}, Z_{n}, i}}{\partial z_{i, \bar{h}}} u_{n} \\
& =O\left(\frac{\varepsilon_{n}^{2}}{\left|\ln \varepsilon_{n}\right|^{p}}\right) .
\end{aligned}
$$

Using (3.5), we find that

$$
b_{i, h, n}=O\left(\varepsilon_{n}^{2}\left|\ln \varepsilon_{n}\right|\right) .
$$

Therefore,

$$
\begin{aligned}
& \sum_{j=1}^{m} \sum_{h=1}^{2} b_{j, h, n}\left(-\delta_{n}^{2} \Delta \frac{\partial P_{\delta_{n}, Z_{n}, j}}{\partial z_{j, h}}\right) \\
& =p \sum_{j=1}^{m} \sum_{h=1}^{2} b_{j, h, n}\left(W_{\delta_{n}, z_{j, n}, a_{\delta_{n}, j}}-a_{\delta_{n}, j}\right)_{+}^{p-1}\left(\frac{\partial W_{\delta_{n}, z_{j, n}, a_{\delta_{n}, j}}}{\partial z_{j, h}}-\frac{\partial a_{\delta_{n}, j}}{\partial z_{j, h}}\right) \\
& =O\left(\sum_{j=1}^{m} \sum_{h=1}^{2} \frac{\varepsilon_{n}^{\frac{2}{p}-1}\left|b_{j, h, n}\right|}{\left|\ln \varepsilon_{n}\right|^{p}}\right) \\
& =O\left(\frac{\varepsilon_{n}^{\frac{2}{p}+1}}{\left|\ln \varepsilon_{n}\right|^{p-1}}\right) \text { in } L^{p}(\Omega) .
\end{aligned}
$$

Thus, we obtain 


$$
L_{\delta_{n}} u_{n}=Q_{\delta_{n}} L_{\delta_{n}} u_{n}+O\left(\frac{\varepsilon_{n}^{\frac{2}{p}+1}}{\left|\ln \varepsilon_{n}\right|^{p-1}}\right)=O\left(\frac{1}{n} \frac{\delta_{n}^{\frac{2}{p}}}{\left|\ln \delta_{n}\right|^{\frac{(p-1)^{2}}{p}}}\right) .
$$

For any fixed $i$, define

Let

$$
\tilde{u}_{i, n}(y)=u_{n}\left(s_{n, i} y+z_{i, n}\right)
$$

$$
\tilde{L}_{n} u=-\Delta u-\sum_{l=1}^{m} p \frac{s_{n, i}^{2}}{\delta_{n}^{2}} \chi_{\Omega_{l}}\left(P_{\delta_{n}, Z_{n}}\left(s_{n, i} y+z_{i, n}\right)-\kappa_{l}-\frac{2 \pi q}{\left|\ln \varepsilon_{n}\right|}\right)_{+}^{p-1} u
$$

Then

Noting that

$$
s_{n, i}^{\frac{2}{p}} \times \frac{\delta_{n}^{2}}{s_{n, i}^{2}}\left\|\tilde{L}_{n} \tilde{u}_{i, n}\right\|_{p}=\left\|L_{\delta_{n}} u_{n}\right\|_{p} .
$$

we find that

$$
\left(\frac{\delta_{n}}{s_{n, i}}\right)^{2}=O\left(\frac{1}{\left|\ln \delta_{n}\right|^{p-1}}\right)
$$

$$
L_{\delta_{n}} u_{n}=o\left(\frac{\delta_{n}^{\frac{2}{p}}}{\left|\ln \delta_{n}\right|^{\frac{(p-1)^{2}}{p}}}\right)
$$

As a result,

$$
\tilde{L}_{n} \tilde{u}_{i, n}=o(1), \quad \text { in } L^{p}\left(\Omega_{n}\right),
$$

where $\Omega_{n}=\left\{y: s_{n, i} y+z_{i, n} \in \Omega\right\}$.

Since $\left\|\tilde{u}_{i, n}\right\|_{\infty}=1$, by the regularity theory of elliptic equations, we may assume that

$$
\tilde{u}_{i, n} \rightarrow u_{i}, \quad \text { in } C_{l o c}^{1}\left(\mathbb{R}^{2}\right)
$$

It is easy to see that

$$
\begin{aligned}
& \sum_{l=1}^{m} \frac{s_{n, i}^{2}}{\delta_{n}^{2}} \chi_{\Omega_{l}}\left(P_{\delta_{n} . Z_{n}}\left(s_{n, i} y+z_{i, n}\right)-\kappa_{l}-\frac{2 \pi q}{\left|\ln \varepsilon_{n}\right|}\right)_{+}^{p-1} \\
& =\frac{s_{n, i}^{2}}{\delta_{n}^{2}}\left(W_{\delta_{n}, z_{i, n}, a_{\delta_{n}, i}}-a_{\delta_{n}, i}+O\left(\frac{s_{n, i}}{\left|\ln \varepsilon_{n}\right|}\right)\right)_{+}^{p-1}+o(1) \\
& \rightarrow w_{+}^{p-1} .
\end{aligned}
$$

Then, by Lemma A.1, we find that $u_{i}$ satisfies

$$
-\Delta u_{i}-p w_{+}^{p-1} u_{i}=0
$$

Now from the Proposition 3.1 in [11], we have 
Since

$$
u_{i}=c_{1} \frac{\partial w}{\partial x_{1}}+c_{2} \frac{\partial w}{\partial x_{2}}
$$

we find that

$$
\int_{\Omega} \Delta\left(\frac{\partial P_{\delta_{n}, Z_{n}, i}}{\partial z_{i, h}}\right) u_{n}=0
$$

$$
\int_{\mathbb{R}^{2}} \phi_{+}^{p-1} \frac{\partial \phi}{\partial z_{h}} u_{i}=0
$$

which, together with (3.7), gives $u_{i}=0$. Thus,

$$
\tilde{u}_{i, n} \rightarrow 0, \quad \text { in } C^{1}\left(B_{L}(0)\right)
$$

for any $L>0$, which implies that $u_{n}=o(1)$ on $\partial B_{L s_{n . i}}\left(z_{i, n}\right)$.

By assumption,

$$
Q_{\delta_{n}} L_{\delta_{n}} u_{n}=0, \quad \text { in } \Omega \backslash \cup_{i=1}^{k} B_{L s_{n, i}}\left(z_{i, n}\right) .
$$

On the other hand, by Lemma A.1, for $j=1, \cdots, m$, we have

Thus, we find that

$$
\left(P_{\delta_{n}, Z_{n}}-\kappa_{j}-\frac{2 \pi q(x)}{\left|\ln \varepsilon_{n}\right|}\right)_{+}=0, \quad x \in \Omega_{j} \backslash B_{L s_{n, j}}\left(z_{j, n}\right) .
$$

$$
-\Delta u_{n}=0, \quad \text { in } \Omega \backslash \cup_{i=1}^{m} B_{L s_{n, i}}\left(z_{i, n}\right) .
$$

However, $u_{n}=0$ on $\partial \Omega$ and $u_{n}=o(1)$ on $\partial B_{L s_{n, i}}\left(z_{i, n}\right), i=1, \cdots, m$. So we have

$$
u_{n}=o(1)
$$

This is a contradiction.

Proposition 3.2. $Q_{\delta} L_{\delta}$ is one to one and onto from $E_{\delta, Z}$ to $F_{\delta, Z}$.

Proof. Suppose that $Q_{\delta} L_{\delta} u=0$. Then, by Lemma 3.1, $u=0$. Thus, $Q_{\delta} L_{\delta}$ is one to one. Next, we prove that $Q_{\delta} L_{\delta}$ is an onto map from $E_{\delta, Z}$ to $F_{\delta, Z}$.

Denote

$$
\tilde{E}=\left\{u: u \in H_{0}^{1}(\Omega), \int_{\Omega} D \frac{\partial P_{\delta, Z, j}}{\partial z_{j, h}} D u=0, j=1, \cdots, m, h=1,2\right\} .
$$

Note that $E_{\delta, Z}=\tilde{E} \cap W^{2, p}(\Omega)$.

For any $\tilde{h} \in F_{\delta, Z}$, by the Riesz representation theorem, there is a unique $u \in H_{0}^{1}(\Omega)$, such that 


$$
\delta^{2} \int_{\Omega} D u D \varphi=\int_{\Omega} \tilde{h} \varphi, \quad \forall \varphi \in H_{0}^{1}(\Omega)
$$

On the other hand, from $\tilde{h} \in F_{\delta, Z}$, we find that $u \in \tilde{E}$. Moreover, by the $L^{p}$-estimate, we deduce that $u \in W^{2, p}(\Omega)$. As a result, $u \in E_{\delta, Z}$. Thus, we see that $Q_{\delta}\left(-\delta^{2} \Delta\right)=-\delta^{2} \Delta$ is an one to one and onto map from $E_{\delta, Z}$ to $F_{\delta, Z}$. On the other hand, $Q_{\delta} L_{\delta} u=h$ is equivalent to

$u=p \delta^{-2}\left(-Q_{\delta} \Delta\right)^{-1}\left[Q_{\delta}\left(\sum_{j=1}^{m} \chi_{\Omega_{j}}\left(P_{\delta, Z}-\kappa_{j}-\frac{2 \pi q(x)}{|\ln \varepsilon|}\right)_{+}^{p-1} u\right)\right]+\delta^{-2}\left(-Q_{\delta} \Delta\right)^{-1} h, \quad u \in E_{\delta, Z}$

It is easy to check that $\delta^{-2}\left(-Q_{\delta} \Delta\right)^{-1}\left[Q_{\delta}\left(\sum_{j=1}^{m} \chi_{\Omega_{j}}\left(P_{\delta, Z}-\kappa_{j}-\frac{2 \pi q(x)}{|\ln \varepsilon|}\right)_{+}^{p-1} u\right)\right]$ is a compact operator in $E_{\delta, Z}$. By the Fredholm alternative, (3.9) is solvable if and only if

$$
u=p \delta^{-2}\left(-Q_{\delta} \Delta\right)^{-1}\left[Q_{\delta}\left(\sum_{j=1}^{m} \chi_{\Omega_{j}}\left(P_{\delta, Z}-\kappa_{j}-\frac{2 \pi q(x)}{|\ln \varepsilon|}\right)_{+}^{p-1} u\right)\right]
$$

has trivial solution, which is true since $Q_{\delta} L_{\delta}$ is a one to one map. Thus the result follows.

Now consider the equation

$$
Q_{\delta} L_{\delta} \omega=Q_{\delta} l_{\delta}+Q_{\delta} R_{\delta}(\omega)
$$

where

$$
l_{\delta}=\sum_{j=1}^{m} \chi_{\Omega_{j}}\left(P_{\delta, Z}-\kappa_{j}-\frac{2 \pi q(x)}{|\ln \varepsilon|}\right)_{+}^{p}-\sum_{j=1}^{m}\left(W_{\delta, z_{j}, a_{\delta, j}}-a_{\delta, j}\right)_{+}^{p}
$$

and

$$
\begin{aligned}
R_{\delta}(\omega)= & \sum_{j=1}^{m} \chi_{\Omega_{j}}\left(P_{\delta, Z}-\kappa_{j}+\omega-\frac{2 \pi q(x)}{|\ln \varepsilon|}\right)_{+}^{p}-\sum_{j=1}^{m} \chi_{\Omega_{j}}\left(P_{\delta, Z}-\kappa_{j}-\frac{2 \pi q(x)}{|\ln \varepsilon|}\right)_{+}^{p} \\
& -\sum_{j=1}^{m} \chi_{\Omega_{j}} p\left(P_{\delta, Z}-\kappa_{j}-\frac{2 \pi q(x)}{|\ln \varepsilon|}\right)_{+}^{p-1} \omega .
\end{aligned}
$$

Using Proposition 3.2, we can rewrite (3.10) as

$$
\omega=G_{\delta} \omega=:\left(Q_{\delta} L_{\delta}\right)^{-1} Q_{\delta}\left(l_{\delta}+R_{\delta}(\omega)\right) .
$$

The next Proposition enables us to reduce the problem of finding a solution for (1.10) to a finite dimensional problem. 
Proposition 3.3. There is an $\delta_{0}>0$, such that for any $\delta \in\left(0, \delta_{0}\right]$ and $Z$ satisfying (3.3), (3.10) has a unique solution $\omega_{\delta} \in E_{\delta, Z}$, with

$$
\left\|\omega_{\delta}\right\|_{\infty}=O\left(\delta|\ln \delta|^{\frac{p-1}{2}}\right) .
$$

Proof. It follows from Lemma A.1 that if $L$ is large enough, $\delta$ is small then

$$
\left(P_{\delta, Z}-\kappa_{j}-\frac{2 \pi q(x)}{|\ln \varepsilon|}\right)_{+}=0, \quad x \in \Omega_{j} \backslash B_{L s_{\delta, j}}\left(z_{j}\right), j=1, \cdots, m .
$$

Let

$$
M=E_{\delta, Z} \cap\left\{\|\omega\|_{\infty} \leq \delta|\ln \delta|^{\frac{p-1}{2}}\right\} .
$$

Then $M$ is complete under $L^{\infty}$ norm and $G_{\delta}$ is a map from $E_{\delta, Z}$ to $E_{\delta, Z}$. We will show that $G_{\delta}$ is a contraction map from $M$ to $M$.

Step $1 . G_{\delta}$ is a map from $M$ to $M$.

For any $\omega \in M$, similar to Lemma A.1, it is easy to prove that for large $L>0, \delta$ small

$$
\left(P_{\delta, Z}+\omega-\kappa_{j}-\frac{2 \pi q(x)}{|\ln \varepsilon|}\right)_{+}=0, \quad \text { in } \Omega_{j} \backslash B_{L s_{\delta, j}}\left(z_{j}\right) .
$$

Note also that for any $u \in L^{\infty}(\Omega)$,

$$
Q_{\delta} u=u \quad \text { in } \Omega \backslash \cup_{j=1}^{m} B_{L s_{\delta, j}}\left(z_{j}\right) .
$$

Therefore, using Lemma A.1, (3.11) and (3.12), we find that for any $\omega \in M$,

$$
Q_{\delta} l_{\delta}+Q_{\delta} R_{\delta}(\omega)=l_{\delta}+R_{\delta}(\omega)=0, \quad \text { in } \Omega \backslash \cup_{j=1}^{m} B_{L s_{\delta, j}}\left(z_{j}\right) .
$$

So, we can apply Lemma 3.1 to obtain

$$
\left\|\left(Q_{\delta} L_{\delta}\right)^{-1}\left(Q_{\delta} l_{\delta}+Q_{\delta} R_{\delta}(\omega)\right)\right\|_{\infty} \leq \frac{C|\ln \delta|^{\frac{(p-1)^{2}}{p}}}{\delta^{\frac{2}{p}}}\left\|Q_{\delta} l_{\delta}+Q_{\delta} R_{\delta}(\omega)\right\|_{p} .
$$

Thus, for any $\omega \in M$, we have

$$
\begin{aligned}
\left\|G_{\delta}(\omega)\right\|_{\infty} & =\left\|\left(Q_{\delta} L_{\delta}\right)^{-1} Q_{\delta}\left(l_{\delta}+R_{\delta}(\omega)\right)\right\|_{\infty} \\
& \leq \frac{C|\ln \delta|^{\frac{(p-1)^{2}}{p}}}{\delta^{\frac{2}{p}}}\left\|Q_{\delta}\left(l_{\delta}+R_{\delta}(\omega)\right)\right\|_{p} .
\end{aligned}
$$

It follows from (3.4) (3.5) that the constant $b_{j, h}$, corresponding to $u \in L^{\infty}(\Omega)$, satisfies

$$
\left|b_{j, h}\right| \leq C|\ln \delta|^{p+1} \sum_{i, \bar{h}} \int_{\Omega}\left|\frac{\partial P_{\delta, Z, i}}{\partial z_{i, \bar{h}}}\right||u| .
$$

Since 


$$
l_{\delta}+R_{\delta}(\omega)=0, \quad \text { in } \Omega \backslash \cup_{j=1}^{m} B_{L s_{\delta, j}}\left(z_{j}\right),
$$

we find that the constant $b_{j, h}$, corresponding to $l_{\delta}+R_{\delta}(\omega)$ satisfies

$$
\begin{aligned}
\left|b_{j, h}\right| & \leq C|\ln \delta|^{p+1} \sum_{i, \bar{h}}\left(\sum_{j=1}^{m} \int_{B_{L s_{\delta, j}}\left(z_{j}\right)}\left|\frac{\partial P_{\delta, Z, i}}{\partial z_{i, \bar{h}}}\right|\left|l_{\delta}+R_{\delta}(\omega)\right|\right) \\
& \leq C \varepsilon^{1-\frac{2}{p}}|\ln \varepsilon|^{p}\left\|l_{\delta}+R_{\delta}(\omega)\right\|_{p} .
\end{aligned}
$$

As a result,

$$
\begin{aligned}
& \left\|Q_{\delta}\left(l_{\delta}+R_{\delta}(\omega)\right)\right\|_{p} \\
\leq & \left\|l_{\delta}+R_{\delta}(\omega)\right\|_{p}+C \sum_{j, h}\left|b_{j, h}\right|\left\|-\delta^{2} \Delta\left(\frac{\partial P_{\delta, Z, j}}{\partial z_{j, h}}\right)\right\|_{p} \\
\leq & C\left\|l_{\delta}\right\|_{p}+C\left\|R_{\delta}(\omega)\right\|_{p} .
\end{aligned}
$$

On the other hand, from Lemma A.1 and (2.12), we can deduce

$$
\begin{aligned}
\left\|l_{\delta}\right\|_{p} & =\left\|\sum_{j=1}^{m} \chi_{\Omega_{j}}\left(P_{\delta, Z}-\kappa_{j}-\frac{2 \pi q(x)}{|\ln \varepsilon|}\right)_{+}^{p}-\sum_{j=1}^{m}\left(W_{\delta, z_{j}, a_{\delta, j}}-a_{\delta, j}\right)_{+}^{p}\right\|_{p} \\
& \leq \sum_{j=1}^{m} \frac{C s_{\delta, j}}{|\ln \varepsilon|}\left\|\left(W_{\delta, z_{j}, a_{\delta, j}}-a_{\delta, j}\right)_{+}^{p-1}\right\|_{p} \\
& =O\left(\frac{\delta^{1+\frac{2}{p}}}{|\ln \delta|^{\frac{p-1}{2}+\frac{1}{p}}}\right) .
\end{aligned}
$$

For the estimate of $\left\|R_{\delta}(\omega)\right\|_{p}$, we have

$$
\begin{aligned}
\left\|R_{\delta}(\omega)\right\|_{p}= & \| \sum_{j=1}^{m} \chi_{\Omega_{j}}\left(P_{\delta, Z}-\kappa_{j}+\omega-\frac{2 \pi q(x)}{|\ln \varepsilon|}\right)_{+}^{p}-\sum_{j=1}^{m} \chi_{\Omega_{j}}\left(P_{\delta, Z}-\kappa_{j}-\frac{2 \pi q(x)}{|\ln \varepsilon|}\right)_{+}^{p} \\
& -\sum_{j=1}^{m} p \chi_{\Omega_{j}}\left(P_{\delta, Z}-\kappa_{j}-\frac{2 \pi q(x)}{|\ln \varepsilon|}\right)_{+}^{p-1} \omega \|_{p} \\
\leq & \sum_{j=1}^{m} C\|\omega\|_{\infty}^{2}\left\|\chi_{\Omega_{j}}\left(P_{\delta, Z}-\kappa_{j}-\frac{2 \pi q(x)}{|\ln \varepsilon|}\right)_{+}^{p-2}\right\|_{p} \\
= & O\left(\frac{\delta^{\frac{2}{p}}\|\omega\|_{\infty}^{2}}{|\ln \delta|^{p-3+\frac{1}{p}}}\right) .
\end{aligned}
$$

Thus, we obtain 


$$
\begin{aligned}
\left\|G_{\delta}(\omega)\right\|_{\infty} & \leq \frac{C|\ln \delta|^{\frac{(p-1)^{2}}{p}}}{\delta^{\frac{2}{p}}}\left(\left\|l_{\delta}\right\|_{p}+\left\|R_{\delta}(\omega)\right\|_{p}\right) \\
& \leq C|\ln \delta|^{\frac{(p-1)^{2}}{p}}\left(\frac{\delta}{|\ln \delta|^{\frac{p-1}{2}+\frac{1}{p}}}+\frac{\|\omega\|_{\infty}^{2}}{|\ln \delta|^{p-3+\frac{1}{p}}}\right) \\
& \leq \delta|\ln \delta|^{\frac{p-1}{2}}
\end{aligned}
$$

Thus, $G_{\delta}$ is a map from $M$ to $M$.

Step 2. $G_{\delta}$ is a contraction map.

In fact, for any $\omega_{i} \in M, i=1,2$, we have

$$
G_{\delta} \omega_{1}-G_{\delta} \omega_{2}=\left(Q_{\delta} L_{\delta}\right)^{-1} Q_{\delta}\left(R_{\delta}\left(\omega_{1}\right)-R_{\delta}\left(\omega_{2}\right)\right)
$$

Noting that

$$
R_{\delta}\left(\omega_{1}\right)=R_{\delta}\left(\omega_{2}\right)=0, \quad \text { in } \Omega \backslash \cup_{j=1}^{m} B_{L s_{\delta, j}}\left(z_{j}\right),
$$

we can deduce as in Step 1 that

$$
\begin{aligned}
\left\|G_{\delta} \omega_{1}-G_{\delta} \omega_{2}\right\|_{\infty} & \leq \frac{C|\ln \delta|^{\frac{(p-1)^{2}}{p}}}{\delta^{\frac{2}{p}}}\left\|R_{\delta}\left(\omega_{1}\right)-R_{\delta}\left(\omega_{2}\right)\right\|_{p} \\
& \leq C|\ln \delta|^{p-1}\left(\frac{\left\|\omega_{1}\right\|_{\infty}}{|\ln \delta|^{p-2}}+\frac{\left\|\omega_{2}\right\|_{\infty}}{|\ln \delta|^{p-2}}\right)\left\|\omega_{1}-\omega_{2}\right\|_{\infty} \\
& \leq C \delta|\ln \delta|^{\frac{p+1}{2}}\left\|\omega_{1}-\omega_{2}\right\|_{\infty} \leq \frac{1}{2}\left\|\omega_{1}-\omega_{2}\right\|_{\infty} .
\end{aligned}
$$

Combining Step 1 and Step 2, we have proved that $G_{\delta}$ is a contraction map from $M$ to $M$. By the contraction mapping theorem, there is an unique $\omega_{\delta} \in M$, such that $\omega_{\delta}=G_{\delta} \omega_{\delta}$. Moreover, it follows from (3.17) that

$$
\left\|\omega_{\delta}\right\|_{\infty} \leq \delta|\ln \delta|^{\frac{p-1}{2}}
$$

\section{Proof of The main Results}

In this section, we will choose $Z$, such that $\sum_{j=1}^{m} P_{\delta, Z, j}+\omega_{\delta}$, where $\omega_{\delta}$ is the map obtained in Proposition 3.3, is a solution of (1.10).

Define

$$
I(u)=\frac{\delta^{2}}{2} \int_{\Omega}|D u|^{2}-\sum_{j=1}^{m} \frac{1}{p+1} \int_{\Omega} \chi_{\Omega_{j}}\left(u-\kappa_{j}-\frac{2 \pi q(x)}{|\ln \varepsilon|}\right)_{+}^{p+1}
$$

and

$$
K(Z)=I\left(P_{\delta, Z}+\omega_{\delta}\right)
$$


It is well known that if $Z$ is a critical point of $K(Z)$, then $\sum_{j=1}^{m} P_{\delta, Z, j}+\omega_{\delta}$ is a solution of (1.10).

In the following, we will prove that $K(Z)$ has a critical point.

Lemma 4.1. We have

$$
K(Z)=I\left(\sum_{j=1}^{m} P_{\delta, Z, j}\right)+O\left(\frac{\varepsilon^{3}}{|\ln \varepsilon|^{p}}\right)
$$

Proof. Recall that

$$
P_{\delta, Z}=\sum_{j=1}^{m} P_{\delta, Z, j}
$$

We have

$$
\begin{aligned}
K(Z)= & I\left(P_{\delta, Z}\right)+\int_{\Omega} \delta^{2} D P_{\delta, Z} D \omega_{\delta}+\frac{\delta^{2}}{2} \int_{\Omega}\left|D \omega_{\delta}\right|^{2} \\
& -\frac{1}{p+1} \sum_{j=1}^{m} \int_{\Omega} \chi_{\Omega_{j}}\left[\left(P_{\delta, Z}+\omega_{\delta}-\kappa_{j}-\frac{2 \pi q(x)}{|\ln \varepsilon|}\right)_{+}^{p+1}-\left(P_{\delta, Z}-\kappa_{j}-\frac{2 \pi q(x)}{|\ln \varepsilon|}\right)_{+}^{p+1}\right] .
\end{aligned}
$$

Using Proposition 3.3 and (3.14), we find

$$
\begin{aligned}
& \int_{\Omega_{j}}\left[\left(P_{\delta, Z}+\omega_{\delta}-\kappa_{j}-\frac{2 \pi q(x)}{|\ln \varepsilon|}\right)_{+}^{p+1}-\left(P_{\delta, Z}-\kappa_{j}-\frac{2 \pi q(x)}{|\ln \varepsilon|}\right)_{+}^{p+1}\right] \\
= & \int_{B_{L s_{\delta, j}}\left(z_{j}\right)}\left[\left(P_{\delta, Z}+\omega_{\delta}-\kappa_{j}-\frac{2 \pi q(x)}{|\ln \varepsilon|}\right)_{+}^{p+1}-\left(P_{\delta, Z}-\kappa_{j}-\frac{2 \pi q(x)}{|\ln \varepsilon|}\right)_{+}^{p+1}\right] \\
= & O\left(\frac{s_{\delta, j}^{2}\left\|\omega_{\delta}\right\|_{\infty}}{|\ln \varepsilon|^{p}}\right)=O\left(\frac{\varepsilon^{3}}{|\ln \varepsilon|^{p}}\right) .
\end{aligned}
$$

On the other hand,

$$
\begin{aligned}
& \delta^{2} \int_{\Omega} D P_{\delta, Z} D \omega_{\delta}=\sum_{j=1}^{m} \int_{\Omega}\left(W_{\delta, z_{j}, a_{\delta, j}}-a_{\delta, j}\right)_{+}^{p} \omega_{\delta} \\
= & \sum_{j=1}^{m} \int_{\cup_{k=1}^{m} B_{s_{\delta, k}}\left(z_{k}\right)}\left(W_{\delta, z_{j}, a_{\delta, j}}-a_{\delta, j}\right)_{+}^{p} \omega_{\delta} \\
= & O\left(\frac{\varepsilon^{3}}{|\ln \varepsilon|^{p}}\right) .
\end{aligned}
$$


Finally, we estimate $\delta^{2} \int_{\Omega}\left|D \omega_{\delta}\right|^{2}$.

Note that

$$
\begin{aligned}
-\delta^{2} \Delta \omega_{\delta}= & \sum_{j=1}^{m} \chi_{\Omega_{j}}\left(P_{\delta, Z}+\omega_{\delta}-\kappa_{j}-\frac{2 \pi q(x)}{|\ln \varepsilon|}\right)_{+}^{p}-\sum_{j=1}^{m}\left(W_{\delta, z_{j}, a_{\delta, j}}-a_{\delta, j}\right)_{+}^{p} \\
& +\sum_{j=1}^{m} \sum_{\bar{h}=1}^{2} b_{j, \bar{h}}\left(-\delta^{2} \Delta \frac{\partial P_{\delta, Z, j}}{\partial z_{j, \bar{h}}}\right),
\end{aligned}
$$

Hence, by (2.12), we have

$$
\begin{aligned}
\delta^{2} \int_{\Omega}\left|D \omega_{\delta}\right|^{2}= & \sum_{j=1}^{m} \int_{\Omega_{j}}\left[\left(P_{\delta, Z}+\omega_{\delta}-\kappa_{j}-\frac{2 \pi q(x)}{|\ln \varepsilon|}\right)_{+}^{p}-\left(W_{\delta, z_{j}, a_{\delta, j}}-a_{\delta, j}\right)_{+}^{p}\right] \omega_{\delta} \\
& +\sum_{j=1}^{m} \sum_{\bar{h}=1}^{2} b_{j, \bar{h}} \int_{\Omega}\left(-\delta^{2} \Delta \frac{\partial P_{\delta, Z, j}}{\partial z_{j, \bar{h}}}\right) \omega_{\delta} \\
= & p \sum_{j=1}^{m} \int_{\Omega_{j}}\left(W_{\delta, z_{j}, a_{\delta, j}}-a_{\delta, j}\right)_{+}^{p-1}\left(\frac{s_{\delta, j}}{|\ln \varepsilon|}+\omega_{\delta}\right) \omega_{\delta}+O\left(\sum_{j=1}^{m} \sum_{\bar{h}=1}^{2} \frac{\varepsilon\left|b_{j, \bar{h}}\right| \mid \omega_{\delta} \|_{\infty}}{|\ln \varepsilon|^{p}}\right) \\
= & O\left(\frac{\varepsilon^{4}}{|\ln \varepsilon|^{p-1}}\right) .
\end{aligned}
$$

So we can obtain that

$$
K(Z)=I\left(\sum_{j=1}^{m} P_{\delta, Z, j}\right)+O\left(\frac{\varepsilon^{3}}{|\ln \varepsilon|^{p}}\right)
$$

Lemma 4.2. We have

$$
\frac{\partial K(Z)}{\partial z_{i, h}}=\frac{\partial}{\partial z_{i, h}} I\left(\sum_{j=1}^{m} P_{\delta, Z, j}\right)+O\left(\frac{\varepsilon^{3}}{|\ln \varepsilon|^{p-1}}\right) .
$$

Proof. First, we have

$$
\begin{aligned}
& \frac{\partial K(Z)}{\partial z_{i, h}}=\left\langle I^{\prime}\left(P_{\delta, Z}+\omega_{\delta}\right), \frac{\partial P_{\delta, Z}}{\partial z_{i, h}}+\frac{\partial \omega_{\delta}}{\partial z_{i, h}}\right\rangle \\
= & \frac{\partial}{\partial z_{i, h}} I\left(P_{\delta, Z}\right)+\left\langle I^{\prime}\left(P_{\delta, Z}+\omega_{\delta}\right), \frac{\partial \omega_{\delta}}{\partial z_{i, h}}\right)^{m} \\
& -\sum_{j=1}^{m} \int_{\Omega_{j}}\left[\left(P_{\delta, Z}+\omega_{\delta}-\kappa_{j}-\frac{2 \pi q(x)}{|\ln \varepsilon|}\right)_{+}^{p}-\left(P_{\delta, Z}-\kappa_{j}-\frac{2 \pi q(x)}{|\ln \varepsilon|}\right)_{+}^{p}\right] \frac{\partial P_{\delta, Z}}{\partial z_{i, h}} .
\end{aligned}
$$


Since $\omega_{\delta} \in E_{\delta, Z}$, we have

$$
\int_{\Omega}\left(W_{\delta, z_{j}, a_{\delta, j}}-a_{\delta, j}\right)_{+}^{p-1}\left(\frac{\partial W_{\delta, z_{j}, a_{\delta, j}}}{\partial z_{j, h}}-\frac{\partial a_{\delta, j}}{\partial z_{j, h}}\right) \omega_{\delta}=0 .
$$

Differentiating the above relation with respect to $z_{i, h}$, we can deduce

$$
\begin{aligned}
& \left\langle I^{\prime}\left(P_{\delta, Z}+\omega_{\delta}\right), \frac{\partial \omega_{\delta}}{\partial z_{i, h}}\right\rangle=\sum_{j=1}^{m} \sum_{\bar{h}=1}^{2} b_{j, \bar{h}} \int_{\Omega}\left(-\delta^{2} \Delta \frac{\partial P_{\delta, Z, j}}{\partial z_{j, \bar{h}}}\right) \frac{\partial \omega_{\delta}}{\partial z_{i, h}} \\
& =\sum_{j=1}^{m} \sum_{\bar{h}=1}^{2} p b_{j, \bar{h}} \int_{\Omega}\left(W_{\delta, z_{j}, a_{\delta, j}}-a_{\delta, j}\right)_{+}^{p-1}\left(\frac{\partial W_{\delta, z_{j}, a_{\delta, j}}}{\partial z_{j, \bar{h}}}-\frac{\partial a_{\delta, j}}{\partial z_{j, \bar{h}}}\right) \frac{\partial \omega_{\delta}}{\partial z_{i, h}} \\
& =O\left(\sum_{j=1}^{m} \sum_{\bar{h}=1}^{2} \frac{\varepsilon\left|b_{j, \bar{h}}\right|}{|\ln \varepsilon|^{p}}\right)=O\left(\frac{\varepsilon^{3}}{|\ln \varepsilon|^{p-1}}\right) .
\end{aligned}
$$

On the other hand, using (3.16) (for the definition of $R_{\delta}(\omega)$, see (3.12)), we obtain

$$
\begin{aligned}
& \sum_{j=1}^{m} \int_{\Omega_{j}}\left[\left(P_{\delta, Z}+\omega_{\delta}-\kappa_{j}-\frac{2 \pi q(x)}{|\ln \varepsilon|}\right)_{+}^{p}-\left(P_{\delta, Z}-\kappa_{j}-\frac{2 \pi q(x)}{|\ln \varepsilon|}\right)_{+}^{p}\right] \frac{\partial P_{\delta, Z, i}}{\partial z_{i, h}} \\
& =\sum_{j=1}^{m} \int_{\Omega_{j}}\left[\left(P_{\delta, Z}+\omega_{\delta}-\kappa_{j}-\frac{2 \pi q(x)}{|\ln \varepsilon|}\right)_{+}^{p}-\left(P_{\delta, Z}-\kappa_{j}-\frac{2 \pi q(x)}{|\ln \varepsilon|}\right)_{+}^{p}\right. \\
& \left.-p\left(P_{\delta, Z}-\kappa_{j}-\frac{2 \pi q(x)}{\kappa|\ln \varepsilon|}\right)_{+}^{p-1} \omega_{\delta}\right] \frac{\partial P_{\delta, Z, i}}{\partial z_{i, h}}+\sum_{j=1}^{m} p \int_{\Omega_{j}}\left[\left(P_{\delta, Z}-\kappa_{j}-\frac{2 \pi q(x)}{|\ln \varepsilon|}\right)_{+}^{p-1}\right. \\
& \left.-\left(W_{\delta, z_{j}, a_{\delta, j}}-a_{\delta, j}\right)_{+}^{p-1}\right] \frac{\partial P_{\delta, Z, i}}{\partial z_{i, h}} \omega_{\delta}+O\left(\frac{s_{\delta, j}^{2}\left\|\omega_{\delta}\right\|_{\infty}}{|\ln \varepsilon|^{p}}\right) \\
& =\int_{\Omega} R_{\delta}\left(\omega_{\delta}\right) \frac{\partial P_{\delta, Z, i}}{\partial z_{i, h}}+\sum_{j=1}^{m} p \int_{\Omega_{j}}\left[\left(P_{\delta, Z}-\kappa_{j}-\frac{2 \pi q(x)}{|\ln \varepsilon|}\right)_{+}^{p-1}-\left(W_{\delta, z_{i}, a_{\delta, i}}-a_{\delta, i}\right)_{+}^{p-1}\right] \frac{\partial P_{\delta, Z, i}}{\partial z_{i, h}} \omega_{\delta} \\
& +O\left(\frac{\varepsilon^{3}}{|\ln \varepsilon|^{p}}\right) \\
& =O\left(\frac{\varepsilon^{3}}{|\ln \varepsilon|^{p-1}}\right) \text {. }
\end{aligned}
$$

Thus, the estimate follows.

Define

$$
c_{\delta, 1}=\frac{C \delta^{2}}{\ln \frac{R}{\varepsilon}}-\frac{\pi \delta^{2} \ln \frac{1}{\varrho}}{\left|\ln \frac{R}{\varepsilon}\right|^{2}}, \quad c_{\delta, 2}=\frac{(C+\eta) \delta^{2}}{\ln \frac{R}{\varepsilon}}
$$


where $\eta>0$ is a small constant and $\varrho>0$ is a fixed small constant. Let

$$
D=\left\{Z=\left(z_{1}, \cdots, z_{m}\right): z_{i} \in \Omega_{\varrho}, i=1, \cdots, m,\left|z_{i}-z_{j}\right| \geq \varrho^{\bar{L}}, i \neq j\right\},
$$

where $\Omega_{\varrho}=\{y: y \in \Omega, d(y, \partial \Omega) \geq \varrho\}$, and $\bar{L}>0$ is a large constant.

Denote $K^{c}=\{Z: Z \in D, K(Z) \leq c\}$. Consider

$$
\left\{\begin{array}{l}
\frac{d Z(t)}{d t}=-D K(Z(t)), t \geq 0 \\
Z(0) \in K^{c_{\delta, 2}}
\end{array}\right.
$$

Lemma 4.3. $Z(t)$ does not leave $D$ before it reaches $K^{c_{\delta, 1}}$.

Proof. Note that

$$
h(x, z)=\frac{1}{2 \pi} \ln \frac{1}{|x-\bar{z}|}+o(1), \quad \frac{\partial h(x, z)}{\partial n}=-\frac{1}{2 \pi|x-\bar{z}|}\left\langle\frac{x-\bar{z}}{|x-\bar{z}|}, n\right\rangle+o(1),
$$

if $z$ is close to $\partial \Omega$, where $n$ is the outward normal unit vector of $\partial\{x: x \in \Omega, d(x, \partial \Omega) \leq$ $d(z, \partial \Omega)\}$ and $\bar{z}$ is the reflection point of $z$ with respect to $\partial \Omega$.

Suppose that there is $t_{0}>0$, such that $Z\left(t_{0}\right)=:\left(z_{1}, \cdots, z_{m}\right) \in \partial D$.

(1) Suppose that there are $i, j \in\{1,2, \cdots, m\}$, such that $i \neq j$ and $\left|z_{i}-z_{j}\right|=\varrho^{\bar{L}}$.

Since $d(\bar{z}, \partial \Omega) \geq \varrho$ and $\bar{z} \notin \Omega$, using (4.3), we get $\left|h\left(z_{j}, z_{j}\right)\right| \leq C^{\prime} \ln \frac{1}{\varrho}$ for any $i, j$, where $C^{\prime}>0$. Thus, we have

$$
\bar{G}\left(z_{i}, z_{j}\right) \geq \ln \frac{1}{\left|z_{i}-z_{j}\right|}-C^{\prime} \ln \frac{1}{\varrho} \geq \bar{L} \ln \frac{1}{\varrho}-C^{\prime} \ln \frac{1}{\varrho} .
$$

Then, by Lemma 4.1 and Proposition A.2, we have

$$
K(Z) \leq \frac{C \delta^{2}}{\ln \frac{R}{\varepsilon}}+\frac{m C^{\prime} \delta^{2} \ln \frac{1}{\varrho}}{\left(\ln \frac{R}{\varepsilon}\right)^{2}}-\frac{\bar{L} \delta^{2} \ln \frac{1}{\varrho}}{\left(\ln \frac{R}{\varepsilon}\right)^{2}}<c_{\delta, 1},
$$

if $\bar{L}>0$ is large.

(2) Suppose that there is $i$, such that $z_{i} \in \partial \Omega_{\varrho}$.

Let $n$ be the outward unit normal of $\partial \Omega_{\varrho}$ at $z_{i}$. We have

$$
\frac{\partial \bar{G}\left(z_{j}, z_{i}\right)}{\partial n}=-\frac{1}{\left|z_{j}-z_{i}\right|}\left\langle\frac{z_{i}-z_{j}}{\left|z_{i}-z_{j}\right|}, n\right\rangle-\frac{\partial g\left(z_{j}, z_{i}\right)}{\partial n}
$$

where $n$ is the outward normal unit vector of $\partial \Omega_{\varrho}$ at $z_{i}$.

On the other hand, if $z_{j} \in \Omega_{\varrho}, j \neq i$, satisfies

$$
\left\langle\frac{z_{i}-z_{j}}{\left|z_{i}-z_{j}\right|}, n\right\rangle<0
$$

then, 
So, we obtain

$$
\left\langle\frac{z_{i}-z_{j}}{\left|z_{i}-z_{j}\right|}, n\right\rangle=O\left(\left|z_{i}-z_{j}\right|\right)
$$

$$
\left\langle\frac{z_{i}-z_{j}}{\left|z_{i}-z_{j}\right|}, n\right\rangle \geq-C\left|z_{i}-z_{j}\right|, \quad \forall j \neq i .
$$

As a result, by Lemma 4.2 and Proposition A.3, we have

$$
\begin{aligned}
\frac{\partial K}{\partial n} \geq & \frac{4 \pi^{2} \delta^{2} \kappa_{i}}{|\ln \varepsilon|\left|\ln \frac{R}{\varepsilon}\right|} \frac{\partial q\left(z_{i}\right)}{\partial n}+\frac{2 \pi \delta^{2} \kappa_{i}^{2}}{\left(\ln \frac{R}{\varepsilon}\right)^{2}} \frac{\partial g\left(z_{i}, z_{i}\right)}{\partial n} \\
& +\sum_{j \neq i}^{m} \frac{2 \pi \delta^{2} \kappa_{i} \kappa_{j}}{\left(\ln \frac{R}{\varepsilon}\right)^{2}} \frac{\partial g\left(z_{j}, z_{i}\right)}{\partial n}-\frac{C \delta^{2}}{\left(\ln \frac{R}{\varepsilon}\right)^{2}} .
\end{aligned}
$$

On the other hand, we derive from (4.3)

$$
\frac{\partial g\left(z_{i}, z_{i}\right)}{\partial n}=\frac{1+o(1)}{2 \varrho}
$$

and

$$
\frac{\partial g\left(z_{j}, z_{i}\right)}{\partial n}=\frac{1+o(1)}{\left|\bar{z}_{i}-z_{j}\right|}\left\langle\frac{\bar{z}_{i}-z_{j}}{\left|\bar{z}_{i}-z_{j}\right|}, n\right\rangle,
$$

where $\bar{z}_{i}$ is the reflection point of $z_{i}$ with respect to $\partial \Omega$.

It is easy to check that if $\left|z_{j}-z_{i}\right| \leq M \varrho$, where $M>0$ is a fixed large constant, then

$$
\left\langle\frac{\bar{z}_{i}-z_{j}}{\left|\bar{z}_{i}-z_{j}\right|}, n\right\rangle \geq 0
$$

So

$$
\frac{\partial K}{\partial n} \geq \frac{2 \pi \delta^{2}}{\left(\ln \frac{R}{\varepsilon}\right)^{2}}\left(\frac{\kappa_{i}^{2}+o(1)}{2 \varrho}-\frac{\kappa_{i} \kappa_{j}+o(1)}{M \varrho}-C\right)>0 .
$$

Therefore, the flow does not leave $D$.

Proof of Theorem 1.6. We will prove that $K(Z)$ has a critical point in $K^{c_{\delta, 2}} \backslash K^{c_{\delta, 1}}$.

Suppose that $K(Z)$ has no critical point in $K^{c_{\delta, 2}} \backslash K^{c_{\delta, 1}}$. Then from Lemma 4.3 that $K^{c_{\delta, 1}}$ is a deformation retract of $K^{c_{\delta, 2}}$.

It is easy to see that $K^{c_{\delta, 2}}=D$ and

$$
\left\{Z: Z \in D,\left|z_{i}-z_{j}\right|=\varrho^{\bar{L}} \text {, for some } i \neq j\right\} \subset K^{c_{\delta, 1}} \text {. }
$$

On the other hand, take $R$ large enough such that $\inf _{\Omega} q \geq-\sum_{i=1}^{m} \frac{(p-1) \kappa_{i}^{2}}{16 m \pi \min _{l}\left\{\kappa_{l}\right\}}-\sum_{i=1}^{m} \frac{\kappa_{i}^{2} g\left(z_{j}, z_{j}\right)}{4 m \pi \min _{l}\left\{\kappa_{l}\right\}}$, then $K(Z) \leq c_{\delta, 1}$ implies that 


$$
-\sum_{j \neq i}^{m} \frac{\pi \delta^{2} \kappa_{i} \kappa_{j} \bar{G}\left(z_{j}, z_{i}\right)}{\left(\ln \frac{R}{\varepsilon}\right)^{2}} \leq-\frac{\pi \delta^{2} \ln \frac{1}{\varrho}}{|\ln \varepsilon|\left|\ln \frac{R}{\varepsilon}\right|},
$$

which implies that there are $i \neq j$, such that

$$
\bar{G}\left(z_{j}, z_{i}\right) \geq c^{\prime} \ln \frac{1}{\varrho}, \quad \text { where } c^{\prime}>0 \text { is a constant. }
$$

So, there is a $\alpha>0$, independent of $\delta$, such that

$$
\left|z_{i}-z_{j}\right| \leq \varrho^{\alpha}
$$

Therefore,

$$
\begin{aligned}
& \left\{Z: Z \in D,\left|z_{i}-z_{j}\right|=\varrho^{\bar{L}} \text {, for some } i \neq j\right\} \\
& \subset K^{c_{\delta, 1}} \subset\left\{Z: Z \in D,\left|z_{i}-z_{j}\right| \leq \varrho^{\alpha}, \text { for some } i \neq j\right\} .
\end{aligned}
$$

Filling the hole $D^{*}=:\left\{Z: Z \in D,\left|z_{i}-z_{j}\right|=\varrho^{\bar{L}}\right.$, for some $\left.i \neq j\right\}$ in $D$, we obtain

$$
\begin{aligned}
& \left\{Z: z_{i} \in \Omega_{\varrho},\left|z_{i}-z_{j}\right| \leq \varrho^{\bar{L}}, \text { for some } i \neq j\right\} \\
& \subset K^{c_{\delta, 1}} \cup D^{*} \subset\left\{Z: z_{i} \in \Omega_{\varrho},\left|z_{i}-z_{j}\right| \leq \varrho^{\alpha}, \text { for some } i \neq j\right\} .
\end{aligned}
$$

Since $K^{c_{\delta, 1}}$ is a deformation retract of $K^{c_{\delta, 2}}$, we find that $K^{c_{\delta, 1}} \cup D^{*}$ is a deformation retract of $K^{c_{\delta, 2}} \cup D^{*}$. On the other hand, $\left\{Z: z_{i} \in \Omega_{\varrho}, z_{i}=z_{j}\right.$, for some $\left.i \neq j\right\}$ is a deformation retract of $\left\{Z: z_{i} \in \Omega_{\varrho},\left|z_{i}-z_{j}\right| \leq \varrho^{\alpha}\right.$, for some $\left.i \neq j\right\}$ if $\varrho>0$ is small. Using (4.5), we see that

$$
\left\{Z: z_{i} \in \Omega_{\varrho}, z_{i}=z_{j}, \text { for some } i \neq j\right\}
$$

is a deformation retract of

$$
\underbrace{\Omega_{\varrho} \times \cdots \times \Omega_{\varrho}}_{m}=K^{c_{\delta, 2}} \cup D^{*} .
$$

This is impossible if $\Omega$ has nontrivial homology.

Thus we get a solution $w_{\delta}$ for (1.10). Let $u_{\varepsilon}=\frac{|\ln \varepsilon|}{2 \pi} w_{\delta}, \delta=\varepsilon\left(\frac{|\ln \varepsilon|}{2 \pi}\right)^{\frac{1-p}{2}}$, it is not difficult to check that $u_{\varepsilon}$ has all the properties listed in Theorem 1.6 and thus the proof of Theorem 1.6 is complete.

Remark 4.4. In the proof of Theorem 1.6, what we actually need is that the following function

$$
\Phi(Z)=\sum_{i=1}^{m} 4 \pi^{2} \kappa_{i} q\left(z_{i}\right)+\sum_{i=1}^{m} \pi \kappa_{i}^{2} g\left(z_{i}, z_{i}\right)-\sum_{j \neq i}^{m} \pi \kappa_{i} \kappa_{j} \bar{G}\left(z_{j}, z_{i}\right)
$$


as well as its small perturbation (in a suitable sense) has a critical point in $D$. Moreover, using the estimates as in Lemma 4.2, it is easy to see that if $\sum_{j=1}^{m} P_{\delta, Z_{\delta}, j}(x)+\omega_{\delta}$ is a solution of (1.10), and $Z_{\delta} \rightarrow Z_{0}$ as $\delta \rightarrow 0$, then $Z_{0}$ is a critical point of $\Phi(Z)$.

Proof of Theorem 1.4. Note that the Kirchhoff-Routh function associated to the vortex dynamics is

$$
\mathcal{W}\left(x_{1}, \cdots, x_{m}\right)=\frac{1}{2} \sum_{i \neq j}^{m} \kappa_{i} \kappa_{j} G\left(x_{i}, x_{j}\right)+\frac{1}{2} \sum_{i=1}^{m} \kappa_{i}^{2} H\left(x_{i}, x_{i}\right)+\sum_{i=1}^{m} \kappa_{i} \psi_{0}\left(x_{i}\right) .
$$

Recall that $h\left(z_{i}, z_{j}\right)=-H\left(z_{i}, z_{j}\right)$, it is easy to check that

$$
\Phi(Z)=-4 \pi^{2} \mathcal{W}(Z)+\sum_{i=1}^{m} \pi \kappa_{i}^{2} \ln R
$$

Hence, $\Phi(Z)$ and $\mathcal{W}(Z)$ possess the same critical points.

By Lemma 4.1, 4.2 and Proposition A.2, A.3, we have

$$
K(Z)=\frac{C \delta^{2}}{\ln \frac{R}{\varepsilon}}+\sum_{i=1}^{m} \frac{\pi(p-1) \delta^{2} \kappa_{i}^{2}}{4\left(\ln \frac{R}{\varepsilon}\right)^{2}}+\frac{\delta^{2}}{|\ln \varepsilon|^{2}} \Phi(Z)+O\left(\frac{\delta^{2} \ln |\ln \varepsilon|}{|\ln \varepsilon|^{3}}\right)
$$

and

$$
\frac{\partial K(Z)}{\partial z_{i, h}}=\frac{\delta^{2}}{|\ln \varepsilon|^{2}} \frac{\partial \Phi(Z)}{\partial z_{i, h}}+O\left(\frac{\delta^{2} \ln |\ln \varepsilon|}{|\ln \varepsilon|^{3}}\right) .
$$

Thus, stable critical point of Kirchhoff-Routh function $\mathcal{W}(Z)$ implies that $K(Z)$ has a critical point. So the result follows.

Now we are in the position to prove Theorem 1.1.

Proof of Theorem 1.1. By Theorem 1.4, we obtain that $u_{\varepsilon}$ is a solution to (1.9).

Set

$$
\begin{gathered}
\mathbf{v}_{\varepsilon}=\left(\nabla\left(u_{\varepsilon}-q\right)\right)^{\perp}, \omega_{\varepsilon}=\nabla \times \mathbf{v}_{\varepsilon}, \\
P_{\varepsilon}=\sum_{j=1}^{m} \frac{1}{p+1} \chi_{\Omega_{j}}\left(u_{\varepsilon}-q-\frac{\kappa_{j}|\ln \varepsilon|}{2 \pi}\right)_{+}^{p+1}-\frac{1}{2}\left|\nabla\left(u_{\varepsilon}-q\right)\right|^{2} .
\end{gathered}
$$

then $\left(\mathbf{v}_{\varepsilon}, P_{\varepsilon}\right)$ forms a stationary solution for problem (1.1).

We now just need to verify

$$
\int_{\Omega} \omega_{\varepsilon} \rightarrow \sum_{j=1}^{m} \kappa_{j}, \text { as } \varepsilon \rightarrow 0
$$


By direct calculations, we find that

$$
\begin{aligned}
\int_{\Omega} \omega_{\varepsilon} & =\sum_{j=1}^{m} \frac{1}{\varepsilon^{2}} \int_{\Omega} \chi_{\Omega_{j}}\left(u_{\varepsilon}-q-\frac{\kappa_{j}|\ln \varepsilon|}{2 \pi}\right)_{+}^{p} \\
& =\sum_{j=1}^{m} \frac{|\ln \varepsilon|^{p}}{(2 \pi)^{p} \varepsilon^{2}} \int_{\Omega_{j}}\left(w_{\delta}-\kappa_{j}-\frac{2 \pi q}{|\ln \varepsilon|}\right)_{+}^{p} \\
& =\sum_{j=1}^{m} \frac{|\ln \varepsilon|^{p}}{(2 \pi)^{p} \varepsilon^{2}} \int_{B_{L s_{\delta, j}\left(z_{j}\right)}}\left(W_{\delta, z_{j}, a_{\delta, j}}-a_{\delta, j}+O\left(\frac{s_{\delta, j}}{|\ln \varepsilon|}\right)\right)_{+}^{p} \\
& =\sum_{j=1}^{m} \frac{s_{\delta, j}^{2}|\ln \varepsilon|^{p}}{(2 \pi)^{p} \varepsilon^{2}}\left(\frac{\delta}{s_{\delta, j}}\right)^{\frac{2 p}{p-1}} \int_{B_{1}(0)} \phi^{p}+o(1) \\
& =\sum_{j=1}^{m} \frac{a_{\delta, j}|\ln \varepsilon|}{\ln \frac{R}{s_{\delta, j}}}+o(1) \\
& \rightarrow \sum_{j=1}^{m} \kappa_{j}, \quad \text { as } \varepsilon \rightarrow 0 .
\end{aligned}
$$

Therefore, the result follows.

Remark 4.5. To regularize point vortices with equi-strength $\kappa$, we do not need $\chi_{\Omega_{j}}$, that is, we just need to consider the following problem

$$
\begin{cases}-\varepsilon^{2} \Delta u=\left(u-q-\frac{\kappa}{2 \pi} \ln \frac{1}{\varepsilon}\right)_{+}^{p}, & x \in \Omega, \\ u=0, & x \in \partial \Omega .\end{cases}
$$

Acknowledgements: D. Cao and Z. Liu were supported by the National Center for Mathematics and Interdisciplinary Sciences, CAS. D. Cao and J. Wei were also supported by CAS Croucher Joint Laboratories Funding Scheme.

\section{ApPendix A. EnERgy EXPANSION}

In this section we will give precise expansions of $I\left(\sum_{j=1}^{m} P_{\delta, Z, j}\right)$ and $\frac{\partial}{\partial z_{i, h}} I\left(\sum_{j=1}^{m} P_{\delta, Z, j}\right)$, which have been used in section 4 .

We always assume that

$$
d\left(z_{j}, \partial \Omega\right) \geq \varrho>0,\left|z_{i}-z_{j}\right| \geq \varrho^{\bar{L}}, \quad i \neq j,
$$

for some small $\varrho>0$ and large $\bar{L}>0$.

Lemma A.1. For $x \in \Omega_{i}, i=1, \cdots, m$, we have

$$
P_{\delta, Z}(x)>\kappa_{i}+\frac{2 \pi q(x)}{|\ln \varepsilon|}, \quad x \in B_{s_{\delta, i}\left(1-T s_{\delta, i}\right)}\left(z_{i}\right),
$$

where $T>0$ is a large constant; while 


$$
P_{\delta, Z}(x)<\kappa_{i}+\frac{2 \pi q(x)}{|\ln \varepsilon|}, \quad x \in \Omega_{i} \backslash B_{s_{\delta, i}\left(1+s_{\delta, i}^{\sigma}\right)}\left(z_{i}\right),
$$

where $\sigma>0$ is a small constant.

Proof. Suppose that $x \in B_{s_{\delta, i}\left(1-T s_{\delta, i}\right)}\left(z_{i}\right)$. It follows from (2.12) and $\phi_{1}^{\prime}(s)<0$ that

$$
\begin{aligned}
& P_{\delta, Z}(x)-\kappa_{i}-\frac{2 \pi q(x)}{|\ln \varepsilon|}=W_{\delta, z_{i}, a_{\delta, i}}(x)-a_{\delta, i}+O\left(\frac{s_{\delta, i}}{|\ln \varepsilon|}\right) \\
= & \frac{a_{\delta, i}}{\left|\phi^{\prime}(1)\right|\left|\ln \frac{R}{s_{\delta, i}}\right|} \phi\left(\frac{\left|x-z_{i}\right|}{s_{\delta, i}}\right)+O\left(\frac{\varepsilon}{|\ln \varepsilon|}\right)>0,
\end{aligned}
$$

if $T>0$ is large. On the other hand, if $x \in \Omega_{i} \backslash B_{s_{\delta, i}^{\tilde{\sigma}}}\left(z_{i}\right)$, where $\tilde{\sigma}>\sigma>0$ is a fixed small constant, then

$$
\begin{aligned}
& P_{\delta, Z}(x)-\kappa_{i}-\frac{2 \pi q(x)}{|\ln \varepsilon|}=\sum_{j=1}^{m} a_{\delta, j} \ln \frac{R}{\left|x-z_{j}\right|} / \ln \frac{R}{s_{\delta, j}}-\kappa_{i}-\frac{2 \pi q(x)}{|\ln \varepsilon|}+o(1) \\
\leq & C \tilde{\sigma}-\kappa_{i}+o(1)<0 .
\end{aligned}
$$

Finally, if $x \in B_{s_{\delta, i}^{\tilde{\sigma}}}\left(z_{i}\right) \backslash B_{s_{\delta, i}\left(1+T s_{\delta, i}^{\tilde{\sigma}}\right)}\left(z_{i}\right)$ for some $i$, then

$$
\begin{aligned}
& P_{\delta, Z}(x)-\kappa_{i}-\frac{2 \pi q(x)}{|\ln \varepsilon|}=W_{\delta, z_{i}, a_{\delta, i}}(x)-a_{\delta, i}+O\left(\frac{s_{\delta, i}^{\tilde{\sigma}}}{\ln \frac{R}{s_{\delta, i}}}\right) \\
= & a_{\delta, i} \frac{\ln \frac{R}{\left|x-z_{i}\right|}}{\ln \frac{R}{s_{\delta, i}}}-a_{\delta, i}+O\left(\frac{s_{\delta, i}^{\tilde{\sigma}}}{\ln \frac{R}{s_{\delta, i}}}\right) \\
\leq & -a_{\delta, i} \frac{\ln \left(1+T s_{\delta, i}^{\tilde{\sigma}}\right)}{\ln \frac{R}{s_{\delta, i}}}+O\left(\frac{s_{\delta, i}^{\tilde{\sigma}}}{\ln \frac{R}{s_{\delta, i}}}\right)<0,
\end{aligned}
$$

if $T>0$ is large. Note that by the choice of $\tilde{\sigma}, B_{s_{\delta, i}\left(1+s_{\delta, i}^{\sigma}\right)}\left(z_{i}\right) \supset B_{s_{\delta, i}\left(1+T s_{\delta, i}^{\tilde{\sigma}}\right)}\left(z_{i}\right)$ for small $\delta$. We therefore derive our conclusion.

Proposition A.2. We have

$$
\begin{aligned}
I\left(P_{\delta, Z}\right)= & \frac{C \delta^{2}}{\ln \frac{R}{\varepsilon}}+\sum_{i=1}^{m} \frac{\pi(p-1) \delta^{2} \kappa_{i}^{2}}{4\left(\ln \frac{R}{\varepsilon}\right)^{2}}+\sum_{i=1}^{m} \frac{4 \pi^{2} \delta^{2} \kappa_{i} q\left(z_{i}\right)}{|\ln \varepsilon|\left|\ln \frac{R}{\varepsilon}\right|}+\sum_{i=1}^{m} \frac{\pi \delta^{2} \kappa_{i}^{2} g\left(z_{i}, z_{i}\right)}{\left(\ln \frac{R}{\varepsilon}\right)^{2}} \\
& -\sum_{j \neq i}^{m} \frac{\pi \delta^{2} \kappa_{i} \kappa_{j} \bar{G}\left(z_{j}, z_{i}\right)}{\left(\ln \frac{R}{\varepsilon}\right)^{2}}+O\left(\frac{\delta^{2} \ln |\ln \varepsilon|}{|\ln \varepsilon|^{3}}\right),
\end{aligned}
$$

where $C$ is a positive constant. 
Proof. Taking advantage of (2.3), we have

$$
\begin{aligned}
& \delta^{2} \int_{\Omega}\left|D P_{\delta, Z}\right|^{2}=\sum_{j=1}^{m} \sum_{i=1}^{m} \int_{\Omega}\left(W_{\delta, z_{j}, a_{\delta, j}}-a_{\delta, j}\right)_{+}^{p} P_{\delta, Z, i} \\
= & \sum_{j=1}^{m} \sum_{i=1}^{m} \int_{B_{s_{\delta, j}}\left(z_{j}\right)}\left(W_{\delta, z_{j}, a_{\delta, j}}-a_{\delta, j}\right)_{+}^{p}\left(W_{\delta, z_{i}, a_{\delta, i}}-\frac{a_{\delta, i}}{\ln \frac{R}{s_{\delta, i}}} g\left(x, z_{i}\right)\right) .
\end{aligned}
$$

First, we estimate

$$
\begin{aligned}
& \int_{B_{s_{\delta, i}}\left(z_{i}\right)}\left(W_{\delta, z_{i}, a_{\delta, i}}-a_{\delta, i}\right)_{+}^{p}\left(W_{\delta, z_{i}, a_{\delta, i}}-\frac{a_{\delta, i}}{\ln \frac{R}{s_{\delta, i}}} g\left(x, z_{i}\right)\right) \\
& =\int_{B_{s_{\delta, i}}\left(z_{i}\right)}\left(W_{\delta, z_{i}, a_{\delta, i}}-a_{\delta, i}\right)^{p+1}+a_{\delta, i} \int_{B_{s_{\delta, i}}\left(z_{i}\right)}\left(W_{\delta, z_{i}, a_{\delta, i}}-a_{\delta, i}\right)^{p} \\
& -\frac{a_{\delta, i}}{\ln \frac{R}{s_{\delta, i}}} \int_{B_{s_{\delta, i}}\left(z_{i}\right)}\left(W_{\delta, z_{i}, a_{\delta, i}}-a_{\delta, i}\right)^{p} g\left(x, z_{i}\right) \\
& =\left(\frac{\delta}{s_{\delta, i}}\right)^{\frac{2(p+1)}{p-1}} s_{\delta, i}^{2} \int_{B_{1}(0)} \phi^{p+1}+a_{\delta, i}\left(\frac{\delta}{s_{\delta, i}}\right)^{\frac{2 p}{p-1}} s_{\delta, i}^{2} \int_{B_{1}(0)} \phi^{p} \\
& -\frac{a_{\delta, i}}{\ln \frac{R}{s_{\delta, i}}}\left(\frac{\delta}{s_{\delta, i}}\right)^{\frac{2 p}{p-1}} g\left(z_{i}, z_{i}\right) s_{\delta, i}^{2} \int_{B_{1}(0)} \phi^{p}+O\left(\frac{s_{\delta, i}^{3}}{|\ln \varepsilon|^{p+1}}\right) \\
& =\frac{\pi(p+1)}{2} \frac{\delta^{2} a_{\delta, i}^{2}}{\left(\ln \frac{R}{s_{\delta, i}}\right)^{2}}+\frac{2 \pi \delta^{2} a_{\delta, i}^{2}}{\ln \frac{R}{s_{\delta, i}}}-\frac{2 \pi \delta^{2} a_{\delta, i}^{2}}{\left(\ln \frac{R}{s_{\delta, i}}\right)^{2}} g\left(z_{i}, z_{i}\right)+O\left(\frac{\varepsilon^{3}}{|\ln \varepsilon|^{p+1}}\right) .
\end{aligned}
$$

Next, for $j \neq i$,

$$
\begin{aligned}
& \int_{B_{s_{\delta, j}}\left(z_{j}\right)}\left(W_{\delta, z_{j}, a_{\delta, j}}-a_{\delta, j}\right)_{+}^{p}\left(W_{\delta, z_{i}, a_{\delta, i}}-\frac{a_{\delta, i}}{\ln \frac{R}{s_{\delta, i}}} g\left(x, z_{i}\right)\right) \\
= & \left(\frac{\delta}{s_{\delta, j}}\right)^{\frac{2 p}{p-1}} \frac{a_{\delta, i}}{\ln \frac{R}{s_{\delta, i}}} \int_{B_{s_{\delta, j}}\left(z_{j}\right)} \phi^{p}\left(\frac{\left|x-z_{j}\right|}{s_{\delta, j}}\right) \bar{G}\left(x, z_{i}\right) \\
= & \left(\frac{\delta}{s_{\delta, j}}\right)^{\frac{2 p}{p-1}} \frac{a_{\delta, i} s_{\delta, j}^{2}}{\ln \frac{R}{s_{\delta, i}}} \int_{B_{1}(0)} \phi^{p}(|x|) \bar{G}\left(z_{j}+s_{\delta, j} x, z_{i}\right) \\
= & \left(\frac{\delta}{s_{\delta, j}}\right)^{\frac{2 p}{p-1}} \frac{a_{\delta, i} s_{\delta, j}^{2}}{\ln \frac{R}{s_{\delta, i}}} \bar{G}\left(z_{j}, z_{i}\right) \int_{B_{1}(0)} \phi^{p}+O\left(\frac{s_{\delta, j}^{3}}{|\ln \varepsilon|^{p+1}}\right) \\
= & \frac{2 \pi \delta^{2} a_{\delta, i} a_{\delta, j}}{\left|\ln \frac{R}{s_{\delta, i}}\right|\left|\ln \frac{R}{s_{\delta, j}}\right|} \bar{G}\left(z_{i}, z_{j}\right)+O\left(\frac{\varepsilon^{3}}{|\ln \varepsilon|^{p+1}}\right) .
\end{aligned}
$$


By Lemma A.1 and (2.12),

$$
\begin{aligned}
& \sum_{j=1}^{m} \int_{\Omega_{j}}\left(P_{\delta, Z}-\kappa_{j}-\frac{2 \pi q(x)}{|\ln \varepsilon|}\right)_{+}^{p+1}=\sum_{j=1}^{m} \int_{B_{L_{\delta}, j}\left(z_{j}\right)}\left(P_{\delta, Z}-\kappa_{j}-\frac{2 \pi q(x)}{\kappa|\ln \varepsilon|}\right)_{+}^{p+1} \\
= & \sum_{j=1}^{m} \int_{B_{L_{s}, j}\left(z_{j}\right)}\left(W_{\delta, z_{j}, a_{\delta, j}}-a_{\delta, j}+O\left(\frac{s_{\delta, j}}{|\ln \varepsilon|}\right)\right)_{+}^{p+1} \\
= & \sum_{j=1}^{m}\left(\frac{\delta}{s_{\delta, j}}\right)^{\frac{2(p+1)}{p-1}} \int_{B_{s_{\delta, j}}\left(z_{j}\right)} \phi^{p+1}\left(\frac{\left|x-z_{j}\right|}{s_{\delta, j}}\right)+O\left(\frac{s_{\delta, j}^{3}}{|\ln \varepsilon|^{p+1}}\right) \\
= & \sum_{j=1}^{m}\left(\frac{\delta}{s_{\delta, j}}\right)^{\frac{2(p+1)}{p-1}} s_{\delta, j}^{2} \int_{B_{1}(0)} \phi^{p+1}+O\left(\frac{s_{\delta, j}^{3}}{|\ln \varepsilon|^{p+1}}\right) \\
= & \sum_{j=1}^{m} \frac{\pi(p+1)}{2} \frac{\delta^{2} a_{\delta, j}^{2}}{\left(\ln \frac{R}{s_{\delta, j}}\right)^{2}}+O\left(\frac{\varepsilon^{3}}{|\ln \varepsilon|^{p+1}}\right) .
\end{aligned}
$$

So, we have proved

$$
\begin{aligned}
I\left(\sum_{i=1}^{m} P_{\delta, Z, j}\right)= & \sum_{i=1}^{m}\left(\frac{\pi(p+1)}{4} \frac{\delta^{2} a_{\delta, i}^{2}}{\left|\ln \frac{R}{s_{\delta, i}}\right|^{2}}+\frac{\pi \delta^{2} a_{\delta, i}^{2}}{\left|\ln \frac{R}{s_{\delta, i}}\right|}-\frac{\pi g\left(z_{i}, z_{i}\right) \delta^{2} a_{\delta, i}^{2}}{\left|\ln \frac{R}{s_{\delta, i}}\right|^{2}}\right) \\
& +\sum_{j \neq i}^{m} \frac{\pi \bar{G}\left(z_{j}, z_{i}\right) \delta^{2} a_{\delta, i} a_{\delta, j}}{\left|\ln \frac{R}{s_{\delta, i}}\right|\left|\ln \frac{R}{s_{\delta, j}}\right|}-\frac{\pi \delta^{2}}{2}\left(\sum_{j=1}^{m} \frac{a_{\delta, j}^{2}}{\left|\ln \frac{R}{s_{\delta, j}}\right|^{2}}\right)+O\left(\frac{\varepsilon^{3}}{|\ln \varepsilon|^{p+1}}\right) .
\end{aligned}
$$

Thus, the result follows from Remark 2.2 .

Proposition A.3. We have

$$
\begin{aligned}
\frac{\partial}{\partial z_{i, h}} I\left(P_{\delta, Z}\right)= & \frac{4 \pi^{2} \delta^{2} \kappa_{i}}{|\ln \varepsilon|\left|\ln \frac{R}{\varepsilon}\right|} \frac{\partial q\left(z_{i}\right)}{\partial z_{i, h}}+\frac{2 \pi \delta^{2} \kappa_{i}^{2}}{\left(\ln \frac{R}{\varepsilon}\right)^{2}} \frac{\partial g\left(z_{i}, z_{i}\right)}{\partial z_{i, h}}-\sum_{j \neq i}^{m} \frac{2 \pi \delta^{2} \kappa_{i} \kappa_{j}}{\left(\ln \frac{R}{\varepsilon}\right)^{2}} \frac{\partial \bar{G}\left(z_{j}, z_{i}\right)}{\partial z_{i, h}} \\
& +O\left(\frac{\delta^{2} \ln |\ln \varepsilon|}{|\ln \varepsilon|^{3}}\right) .
\end{aligned}
$$

Proof. Direct computation yields that

$$
\begin{aligned}
\frac{\partial}{\partial z_{i, h}} I\left(P_{\delta, Z}\right) & =\delta^{2} \int_{\Omega} D P_{\delta, Z} D \frac{\partial P_{\delta, Z}}{\partial z_{i, h}}-\sum_{j=1}^{m} \int_{\Omega_{j}}\left(P_{\delta, Z}-\kappa_{j}-\frac{2 \pi q(x)}{|\ln \varepsilon|}\right)_{+}^{p} \frac{\partial P_{\delta, Z}}{\partial z_{i, h}} \\
& =\sum_{l=1}^{m} \sum_{j=1}^{m} \int_{B_{L s_{\delta, j}}\left(z_{j}\right)}\left[\left(W_{\delta, z_{j}, a_{\delta, j}}-a_{\delta, j}\right)_{+}^{p}-\left(P_{\delta, Z}-\kappa_{j}-\frac{2 \pi q(x)}{|\ln \varepsilon|}\right)_{+}^{p}\right] \frac{\partial P_{\delta, Z, l}}{\partial z_{i, h}} .
\end{aligned}
$$


Using (2.12), Lemma A.1 and Remark 2.2, we find that

$$
\begin{aligned}
& \int_{B_{L s_{\delta, i}}\left(z_{i}\right)}\left[\left(W_{\delta, z_{i}, a_{\delta, i}}-a_{\delta, i}\right)_{+}^{p}-\left(P_{\delta, Z}-\kappa_{i}-\frac{2 \pi q(x)}{|\ln \varepsilon|}\right)_{+}^{p}\right] \frac{\partial P_{\delta, Z, i}}{\partial z_{i, h}} \\
& =\int_{B_{s_{\delta, i}\left(1+s_{\delta, i}^{\sigma}\right)}\left(z_{i}\right)}\left[\left(W_{\delta, z_{i}, a_{\delta, i}}-a_{\delta, i}\right)_{+}^{p}-\left(P_{\delta, Z}-\kappa_{i}-\frac{2 \pi q(x)}{|\ln \varepsilon|}\right)_{+}^{p}\right] \frac{\partial P_{\delta, Z, i}}{\partial z_{i, h}} \\
& =p \int_{B_{s_{\delta, i}}\left(z_{i}\right)}\left(W_{\delta, z_{i}, a_{\delta, i}}-a_{\delta, i}\right)_{+}^{p-1}\left[\frac{2 \pi}{|\ln \varepsilon|}\left\langle D q\left(z_{i}\right), x-z_{i}\right\rangle+\frac{a_{\delta, i}}{\ln \frac{R}{s_{\delta, i}}}\left\langle D g\left(z_{i}, z_{i}\right), x-z_{i}\right\rangle\right. \\
& \left.-\sum_{j \neq i}^{m} \frac{a_{\delta, j}}{\ln \frac{R}{s_{\delta, j}}}\left\langle D \bar{G}\left(z_{i}, z_{j}\right), x-z_{i}\right\rangle\right] \frac{\partial P_{\delta, Z, i}}{\partial z_{i, h}}+O\left(\frac{\varepsilon^{2+\sigma}}{|\ln \varepsilon|^{p+1}}\right) \\
& =-\frac{p \delta^{2} a_{\delta, i}}{\left|\phi^{\prime}(1)\right|\left|\ln \frac{R}{s_{\delta, i}}\right|}\left(\frac{2 \pi}{|\ln \varepsilon|} \frac{\partial q\left(z_{i}\right)}{\partial z_{i, h}}+\frac{a_{\delta, i}}{\ln \frac{R}{s_{\delta, i}}} \frac{\partial g\left(z_{i}, z_{i}\right)}{\partial z_{i, h}}-\sum_{j \neq i}^{m} \frac{a_{\delta, j}}{\ln \frac{R}{s_{\delta, j}}} \frac{\partial \bar{G}\left(z_{i}, z_{j}\right)}{\partial z_{i, h}}\right) \\
& \times \int_{B_{1}(0)} \phi^{p-1}(|x|) \phi^{\prime}(|x|) \frac{x_{h}^{2}}{|x|}+O\left(\frac{\varepsilon^{2+\sigma}}{|\ln \varepsilon|^{p+1}}\right) \\
& =\frac{4 \pi^{2} \delta^{2} a_{\delta, i}}{|\ln \varepsilon|\left|\ln \frac{R}{s_{\delta, i}}\right|} \frac{\partial q\left(z_{i}\right)}{\partial z_{i, h}}+\frac{2 \pi \delta^{2} a_{\delta, i}^{2}}{\left(\ln \frac{R}{s_{\delta, i}}\right)^{2}} \frac{\partial g\left(z_{i}, z_{i}\right)}{\partial z_{i, h}}-\sum_{j \neq i} \frac{2 \pi \delta^{2} a_{\delta, i} a_{\delta, j}}{\left|\ln \frac{R}{s_{\delta, j}}\right|\left|\ln \frac{R}{s_{\delta, i}}\right|} \frac{\partial \bar{G}\left(z_{i}, z_{j}\right)}{\partial z_{i, h}}+O\left(\frac{\varepsilon^{2+\sigma}}{|\ln \varepsilon|^{p+1}}\right),
\end{aligned}
$$

since

$$
\int_{B_{1}(0)} \phi^{p-1}(|x|) \phi^{\prime}(|x|) \frac{x_{h}^{2}}{|x|}=-\frac{2 \pi}{p}\left|\phi^{\prime}(1)\right| .
$$

On the other hand, for $l \neq i$, from (2.12), we have

$$
\begin{aligned}
& \int_{B_{L s_{\delta, j}}\left(z_{j}\right)}\left[\left(W_{\delta, z_{j}, a_{\delta, j}}-a_{\delta, j}\right)_{+}^{p}-\left(P_{\delta, Z}-\kappa_{j}-\frac{2 \pi q(x)}{|\ln \varepsilon|}\right)_{+}^{p}\right] \frac{\partial P_{\delta, Z, l}}{\partial z_{i, h}} \\
= & \int_{B_{L s_{\delta, j}}\left(z_{j}\right)}\left[\left(W_{\delta, z_{j}, a_{\delta, j}}-a_{\delta, j}\right)^{p-1} \frac{s_{\delta, j}}{|\ln \varepsilon|}\right] \times \frac{C}{\ln \frac{R}{s_{\delta, l}}} \\
= & O\left(\frac{\varepsilon^{3}}{|\ln \varepsilon|^{p+1}}\right) .
\end{aligned}
$$

Thus, the result follows.

\section{REFERENCES}

[1] A. Ambrosetti and M. Struwe, Existence of steady vortex rings in an ideal fluid, Arch. Rational Mech. Anal., 108(1989), 97-109. 
[2] A. Ambrosetti and J. Yang, Asymptotic behaviour in planar vortex theory, Atti Accad. Naz. Lincei Cl. Sci. Fis. Mat. Natur. Rend. Lincei (9) Mat. Appl., 1(1990), 285-291.

[3] V.I. Arnold and B.A. Khesin, Topological methods in hydrodynamics, Applied Mathematical Sciences, Vol. 125. Springer, New York, 1998.

[4] T.V. Badiani, Existence of steady symmetric vortex pairs on a planar domain with an obstacle, Math. Proc. Cambridge Philos. Soc., 123(1998), 365-384.

[5] T.Bartsch, A.Pistoia and T.Weth, N-vortex equilibria for ideal fluids in bounded planar domains and new nodal solutions of the sinh-Poisson and the Lane-Emden-Fowler equations, Comm.Math.Phys., 297(2010), 653-686.

[6] M.S. Berger and L.E. Fraenkel, Nonlinear desingularization in certain free-boundary problems, Comm. Math. Phys., 77(1980), 149-172.

[7] G.R. Burton, Vortex rings in a cylinder and rearrangements, J. Diff. Equat., 70(1987), 333-348.

[8] L. Caffarelli and A. Friedman, Asymptotic estimates for the plasma problem, Duke Math. J., 47(1980), $705-742$.

[9] D.Cao and T.Küpper, On the existence of multi-peaked solutions to a semilinear Neumann problem, Duke Math. J., 97(1999), 261-300.

[10] D. Cao, S. Peng and S. Yan, Multiplicity of solutions for the plasma problem in two dimensions, Adv. Math., 225(2010), 2741-2785.

[11] E.N. Dancer and S. Yan, The Lazer-McKenna conjecture and a free boundary problem in two dimensions, J. London Math. Soc., 78(2008), 639-662.

[12] L.E. Fraenkel and M.S. Berger, A global theory of steady vortex rings in an ideal fluid, Acta Math., 132(1974), 13-51.

[13] F. Flucher and J. Wei, Asymptotic shape and location of small cores in elliptic free-boundary problems, Math. Z., 228(1998), 638-703.

[14] W. Kulpa, The Poincaré-Miranda theorem, The Amer. Math. Monthly, 104(1997), 545-550.

[15] G. Li, S. Yan and J. Yang, An elliptic problem related to planar vortex pairs, SIAM J. Math. Anal., 36(2005), 1444-1460.

[16] Y. Li and S. Peng, Multiple solutions for an elliptic problem related to vortex pairs, J. Diff. Equat., 250(2011), 3448-3472.

[17] C.C.Lin, On the motion of vortices in two dimension - I. Existence of the Kirchhoff-Routh function, Proc. Natl. Acad. Sci. USA, 27(1941), 570-575.

[18] C. Miranda, Un'osservazione su un teorema di Brouwer., Boll. Un. Mat. Ital., 3(1940), 5-7.

[19] C. Marchioro and M. Pulvirenti, Euler evolution for singular initial data and vortex theory, Comm. Math. Phys., 91(1983), 563-572.

[20] W.-M. Ni, On the existence of global vortex rings, J. Anal. Math., 37(1980), 208-247.

[21] J. Norbury, Steady planar vortex pairs in an ideal fluid, Comm. Pure Appl. Math., 28(1975), 679-700.

[22] D. Smets and J. Van Schaftingen, Desingulariation of vortices for the Euler equation, Arch. Rational Mech. Anal., 198(2010), 869-925.

[23] B. Turkington, On steady vortex flow in two dimensions. I, II, Comm. Partial Diff. Equat., 8(1983), 999-1030, 1031-1071.

[24] J. Yang, Existence and asymptotic behavior in planar vortex theory, Math. Models Methods Appl. Sci., 1(1991), 461-475.

[25] J. Yang, Global vortex rings and asymptotic behaviour, Nonlinear Anal., 25(1995), 531-546. 
Institute of Applied Mathematics, Chinese Academy of Science, Beijing 100190, P.R. CHINA

E-mail address: dmcao@amt.ac.cn

Institute of Applied Mathematics, Chinese Academy of Science, Beijing 100190, P.R. CHINA

E-mail address: liuzy@amss.ac.cn

Department of Mathematics, The Chinese University of Hong Kong, Shatin, N.T., Hong KONG

E-mail address: wei@math.cuhk.edu.hk 